Article

\title{
Tough and Low Friction Polyvinyl Alcohol Hydrogels Loaded with Anti-inflammatories for Cartilage Replacement
}

\author{
Andreia Sofia Oliveira ${ }^{1,2}{ }^{(1)}$, Sara Schweizer ${ }^{3}$, Pedro Nolasco ${ }^{1,4}$, Isabel Barahona ${ }^{4}$, \\ Jorge Saraiva ${ }^{5}\left(\mathbb{D}\right.$, , Rogério Colaço $^{2, * \mathbb{C}}$ and Ana Paula Serro ${ }^{1,4, * \mathbb{C}}$ \\ 1 Centro de Química Estrutural (CQE), Instituto Superior Técnico, Universidade de Lisboa, Av. Rovisco Pais 1, \\ 1049-001 Lisbon, Portugal; andreia.oliveira@tecnico.ulisboa.pt (A.S.O.); \\ pedro.nolasco@tecnico.ulisboa.pt (P.N.) \\ 2 Instituto de Engenharia Mecânica (IDMEC), Instituto Superior Técnico, Universidade de Lisboa, \\ Av. Rovisco Pais 1, 1049-001 Lisbon, Portugal \\ 3 Faculty of Applied Chemistry, Reutlingen University, Alteburgstraße 150, 72762 Reutlingen, Germany; \\ sara_katharina.schweizer@student.reutlingen-university.de \\ 4 Centro de Investigação Interdisciplinar Egas Moniz (CiiEM), Instituto Universitário Egas Moniz, \\ Quinta da Granja, Monte de Caparica, 2829-511 Caparica, Portugal; ibarahona@egasmoniz.edu.pt \\ 5 QOPNA \& LAQV-REQUIMTE, Departamento de Química, Universidade de Aveiro, \\ Campus Universitário de Santiago, 3810-193 Aveiro, Portugal; jorgesaraiva@ua.pt \\ * Correspondence: rogerio.colaco@tecnico.ulisboa.pt (R.C.); anapaula.serro@tecnico.ulisboa.pt (A.P.S.); \\ Tel.: +351-218-419-240 (A.P.S.)
}

Received: 2 January 2020; Accepted: 15 March 2020; Published: 23 March 2020

check for updates

\begin{abstract}
The development of new materials that mimic cartilage and its function is an unmet need that will allow replacing the damaged parts of the joints, instead of the whole joint. Polyvinyl alcohol (PVA) hydrogels have raised special interest for this application due to their biocompatibility, high swelling capacity and chemical stability. In this work, the effect of post-processing treatments (annealing, high hydrostatic pressure (HHP) and gamma-radiation) on the performance of PVA gels obtained by cast-drying was investigated and, their ability to be used as delivery vehicles of the anti-inflammatories diclofenac or ketorolac was evaluated. HHP damaged the hydrogels, breaking some bonds in the polymeric matrix, and therefore led to poor mechanical and tribological properties. The remaining treatments, in general, improved the performance of the materials, increasing their crystallinity. Annealing at $150{ }^{\circ} \mathrm{C}$ generated the best mechanical and tribological results: higher resistance to compressive and tensile loads, lower friction coefficients and ability to support higher loads in sliding movement. This material was loaded with the anti-inflammatories, both without and with vitamin E (Vit.E) or Vit.E + cetalkonium chloride (CKC). Vit.E + CKC helped to control the release of the drugs which occurred in $24 \mathrm{~h}$. The material did not induce irritability or cytotoxicity and, therefore, shows high potential to be used in cartilage replacement with a therapeutic effect in the immediate postoperative period.
\end{abstract}

Keywords: cartilage replacement; polyvinyl alcohol hydrogel; high hydrostatic pressure; annealing; gamma radiation; controlled drug release; diclofenac; ketorolac

\section{Introduction}

The population aging and the increasing prevalence of obesity have raised the onset of articular cartilage $(\mathrm{AC})$ diseases and therefore increased the need to develop new biomaterials that mimic cartilage tissue and its function. AC is a connective tissue present on the articular surfaces that, 
although tough and flexible, may suffer wear over the years or be damaged by injury or accidents. Due to its avascular and aneural structure, AC is not capable of developing a normal inflammatory response, and its ability for self-repair is quite limited [1,2]. Depending on the nature and extent of the damage, different strategies may be followed to recover its function. These may go from simple and non-invasive procedures, such as the use of physiotherapy to strengthen the surrounding muscles and anti-inflammatory drugs to reduce pain and swelling, to resurfacing or even total joint replacement, when the patient's symptoms are already so severe that there is no alternative but surgical intervention [2]. In the latter case, and in a more conservative perspective, some clinicians are opting to use tissue engineering and regenerative medicine products or synthetic cartilage materials to substitute the damaged parts rather than replace the entire joint [3,4]. Synthetic materials are easily controllable and reproducible and minimize the risk of infections, as they do not contain substances derived from human or animal tissues. They have been used in a few commercial products [4]. For example, SaluCartilage ${ }^{\mathrm{TM}}$ (SaluMedica), a cylindrical device made of polyvinyl alcohol (PVA) hydrogel that mimics the swelling of human cartilage, shows no evidence of an inflammatory reaction or osteolysis and leads to a clear improvement of chondral defects. Cartiva ${ }^{\circledR}$ synthetic cartilage implants are also made of PVA. They are recommended to be used in the first metatarsophalangeal joint, allowing to maintain the normal movement of the articulation. Other materials, such as biphasic polyurethane (e.g., Chondrocushion, Advanced Bio Surfaces) or polyglycolic acid-hyaluronan (Chondrotissue ${ }^{\circledR}$, Biotissue) have also been used. In general, these implants do not require the destruction or removal of healthy tissue from the patient. The materials are placed into pre-drilled holes to resurface the damaged cartilage area.

Presently, the most promising biomaterials for this purpose are hydrogels-crosslinked networks of hydrophilic polymers with a high capacity to absorb water and other molecules-which, besides being biocompatible, can be tailored to combine exceptional lubricity with suitable mechanical properties, as in natural cartilage [2,5]. Several possibilities can be found in literature, including hydrogels based on polyvinyl pyrrolidone, polyethylene glycol, polyhydroxyethyl methacrylate, silicon rubber, gelatin and polyacrylamide [3,6]. Currently, one of the most studied is PVA [7-9]. Since it is highly soluble in water, it needs to be crosslinked to be used as a cartilage substitute. The degree of crosslinking determines the swelling capacity of the material and, thus, its physical, chemical, and diffusional properties, which will ultimately define its biological function [9]. PVA hydrogels may be produced by a variety of methods, namely freeze-thawing (FT), cast drying (CD), irradiation, or injection molding [10]. One of the main issues relative to these materials is the lack of fracture strength and elastic modulus that impairs them to support the expected load [2].

Numerous approaches have been attempted to strengthen and toughen these type of biomaterials, including the incorporation of nano/microparticles (e.g., silica, hydroxyapatite, and polydopamine) [11-13], chemical crosslinkers (e.g., glyoxal, glutaraldehyde, acetaldehyde, or other mono-aldehydes) [14-16], other polymers (e.g., poly(acrylic) acid and hyaluronic acid) [17,18], or differentiated structures (e.g., fibers, nanosheets, and meshes) [19-22]. Chemical crosslinkers are often toxic, inevitably affecting the biocompatibility [20]. Incorporation of differentiated structures may entail adhesion problems and generally require procedures to promote mechanical interlocking or chemical bonding between the structures and the hydrogel [19].

The simplest and safest way to improve the mechanical properties of the hydrogels is by setting adequate preparation conditions that allow a high degree of crosslinking, avoiding the need to add external agents. Suzuki et al. [23] studied the relationship between swelling behavior and mechanical properties of PVA CD gels produced at different temperatures and relative humidity and concluded that such parameters strongly influenced the structure of the gels obtained, i.e., the size, number, and distribution of microcrystallites, determining the material's behavior [24]. In particular, they observed that it is possible to greatly enhance the mechanical strength of the polymers by increasing the relative humidity up to $80 \%$ while keeping the gelation temperature at $60{ }^{\circ} \mathrm{C}$, but this leads to an accentuated reduction in their water absorption capacity [23]. High hydrostatic pressure (HHP) has also been used to prepare hydrogels with improved mechanical properties. Negishi et al. [25] produced 
antithrombotic PVA-heparin hybrid gels using HHP (980 MPa) or FT (one cycle) and observed that the mechanical strength of the ones treated with HHP was much higher. Lian et al. [26] investigated the impact of pre-treating PVA-chitosan solutions with HHP (200-600 MPa) before film formation and realized that it promoted the development of denser 3D networks, with higher tensile strength and elongation at break. Post-preparation treatments with physical agents are also reported in the literature as useful tools for obtaining more robust PVA gels. Concerning heat, it is known that the thermal annealing between the glass transition temperature $\left(T_{g}\right)$ and the melting temperature allows the polymer chains to have sufficient mobility to align and form larger crystallites $[27,28]$. Increasing the annealing temperature leads to the rise of the crystallinity degree and $T_{g}$ (both measurements of crosslinking degree), resulting in more resistant materials [28]. Radiation exposure may also lead to additional crosslinking, changing the overall properties of the gels. Mondino et al. [29] evaluated the effect of gamma radiation on the performance of PVA films prepared by CD and found that tensile strength reached its maximum in samples irradiated with $80 \mathrm{kGy}$. Sasaki et al. [30] concluded that such radiation might also improve friction and wear behavior of PVA gels at physiological temperature. In turn, the effect of post-processing hydrogels by HHP was never studied.

As referred above, hydrogels exhibit a high water absorption capacity, making them ideal platforms for encapsulating hydrophilic drugs and releasing them locally [31]. In addition, hydrogel-based systems can also protect drugs from rapid clearance, improve therapeutic effects due to their ability to control drug release, and reduce side effects as they require lower doses than those used in the conventional method of drug administration (i.e., via systemic route). In the last years, various researchers have explored the possibility of using hydrogels as drug delivery vehicles for articular cartilage repair and osteoarthritis therapy $[32,33]$. Such investigations focused essentially on the local release of growth factors and viral and non-viral vectors [33]. The release of small molecules, such as anti-inflammatory drugs, to treat osteoarthritis, has only been addressed in a reduced number of scientific studies using scaffolds (e.g., [34], or injectable systems with drug-loaded microspheres [35-37]. Non-steroidal anti-inflammatory drugs are effective anti-inflammatory, analgesic, and antipyretic medicines that may be included, with advantages, in cartilage replacement materials, to help in the postoperative recovery period. They inhibit the activity of cyclooxygenase enzymes, which take part in the synthesis of prostaglandins and thromboxane, important mediators of inflammation, pain, and fever [38]. As far as the authors know, to date, there are no studies in the literature reporting the development of potential cartilage substitutes containing anti-inflammatories.

To maintain effective concentrations of anti-inflammatories in the tissues, it is necessary to ensure the controlled release of the drug from hydrogels over an appropriate period. The release kinetics of the drug depends on the mesh size of the material, as it controls the steric interactions between the drug molecules and the polymer network and, therefore, the rate of drug diffusion [31]. Furthermore, drugs can interact with the polymeric chains via a range of mechanisms, including covalent bonding, electrostatic interactions, and hydrophobic associations [31]. The most straightforward and inexpensive method of loading hydrogels is to soak them in the drug solution. However, this strategy usually leads to a rapid release of the drug and an initial burst [39]. Several approaches can be used to improve the release behavior, e.g., incorporation into the polymer matrix of ligands or functional monomers with special affinity for the drug, drug-loaded nanoparticles that release the drug in a controlled manner, molecules which act as diffusion barriers to the drug molecules (e.g., vitamin E) or surfactants that interact with the drugs [40-43].

The present work aimed to develop crosslinked PVA hydrogels for cartilage replacement, with high mechanical strength and suitable tribological properties, that can be used simultaneously for the local release of anti-inflammatories (diclofenac (DFN) or ketorolac (KTL)), in the immediate postoperative period. CD gels were produced in carefully chosen temperature and humidity conditions and submitted to annealing, HHP treatment, or gamma radiation. Their properties (swelling capacity, wettability, morphology, mechanical performance, and friction behavior) were evaluated and compared. The most promising material was loaded with DFN or KTL, and drug release 
profiles were obtained under sink conditions. The effect of pre-loading the hydrogel with vitamin $\mathrm{E}$ and the surfactant cetalkonium chloride on drug release was investigated. Finally, irritability and cytotoxicity tests were also performed.

\section{Materials and Methods}

\subsection{Hydrogels Preparation}

Polyvinyl alcohol (PVA) aqueous solutions (13.5\% w/w) were prepared by dissolving the PVA powder (Kuraray Poval' ${ }^{\mathrm{TM}}$ 28-99 from Kuraray (Tokyo, Japan) - with a hydrolysis degree of 99-99.8 $\mathrm{mol} \%$ and an average molecular weight of 145,000 Da) - in distilled and deionized (DD) water at $95{ }^{\circ} \mathrm{C}$, for a period of $20 \mathrm{~h}$. DD water $(\rho \geq 18 \mathrm{M} \Omega \cdot \mathrm{cm})$ was obtained using a Milli-Q ${ }^{\circledR}$ Integral 3 water purification system (Millipore, Darmstadt, Germany). During the dissolution process, the solutions were gently agitated several times, to ensure homogenization and avoid gas bubble formation. The solutions were then poured carefully into preheated (at $95{ }^{\circ} \mathrm{C}$ ) glass Petri dishes, cooled to room temperature $\left(8 \mathrm{~h}\right.$ ), and dried for seven days at $60^{\circ} \mathrm{C}$ with $80 \%$ of relative humidity to obtain the samples from now on referred to as "Control".

The resulting materials were additionally submitted to different treatments:

- Annealing at $100{ }^{\circ} \mathrm{C}$ or $150{ }^{\circ} \mathrm{C}$ for $30 \mathrm{~min}$ (samples A100 and A150, respectively).

- High hydrostatic pressure at $70{ }^{\circ} \mathrm{C}$ and $600 \mathrm{MPa}$ for $10 \mathrm{~min}$ (samples HHP). Samples were previously packed and sealed in polyamide/polyethylene vacuum bags ( $90 \mu$, Penta Ibérica - Soc. Ibérica de Embalagens, Lda, Torres Vedras, Portugal) filled with $200 \mathrm{~mL}$ of DD water and pre-heated at $70{ }^{\circ} \mathrm{C}$ for $10 \mathrm{~min}$. Then, they were placed inside a polytetrafluoroethylene insulation vessel pre-filled with water at the same temperature and pressurized in a Hiperbaric 55 equipment (Burgos, Spain). The rate of pressurization till the plateau of $600 \mathrm{MPa}$ was $250 \mathrm{MPa} \cdot \mathrm{min}^{-1}$, while depressurization was instantaneous.

- Gamma radiation with a dose of $75 \mathrm{kGy}$ (samples GR). The irradiation was done at room temperature using ${ }^{60} \mathrm{Co}$ as a source of $\gamma$-rays.

After treatments, all samples were washed in DD water (periodically changed) for $48 \mathrm{~h}$ and the resulting samples were stored hydrated in sealed containers, at room temperature, till being used. Prior to each characterization test, the samples were cut with the appropriate dimensions, rinsed, carefully wiped with absorbent paper, and soaked in a fresh DD water or phosphate buffered saline (PBS) solution for at least $24 \mathrm{~h}$.

Overall, each hydrogel type was prepared at least twice in triplicate. The average thickness of the samples, in their swollen state, was $1.7 \pm 0.3 \mathrm{~mm}$.

\subsection{Hydrogels Characterization}

\subsubsection{Microstructural Analysis}

Scanning electron microscopy (SEM) observation of the materials was made at $3 \mathrm{kV}$ with a Field Emission Gun - Scanning Electron Microscope JSM-7001F from JEOL (Tokyo, Japan). To access the surface and cross-section morphologies, discs of each type of sample (diameter $12 \mathrm{~mm}$ ) were placed in a $-80{ }^{\circ} \mathrm{C}$ freezer for $30 \mathrm{~min}$ and subsequently lyophilized for $48 \mathrm{~h}$ in a freeze dryer (LBFD-A21, Labtron, Fleet, Hampshire, UK). For the cross-section evaluation, samples were previously immersed in liquid nitrogen for $10 \mathrm{~s}$ and immediately sectioned in half. Before SEM observations, samples were coated with $20 \mathrm{~nm}$ of a conductive layer of Au:Pd (20:80) using a turbo-pumped sputter coater (Q150T ES, Quorum Technologies, Lewes, UK). 


\subsubsection{Water Uptake}

Discs of hydrated samples (diameter $10 \mathrm{~mm}$ ) were weighed in a semi-micro analytical balance (Discovery DV215CD, Ohaus Corporation, Parsippany, NJ, USA) and then dried at $60^{\circ} \mathrm{C}$ until achieving constant weight. The equilibrium water content (EWC) was estimated using the following equation:

$$
\operatorname{EWC}(\%)=\frac{W_{S}-W_{D}}{W_{S}} \times 100
$$

where $W_{S}$ is the initial weight of the swollen samples and $W_{D}$ is the weight of the dry samples. For each hydrogel, three independent measurements were performed.

\subsubsection{Wettability}

The wettability of the hydrogels was studied using the captive bubble method. Rectangular strips of the hydrated materials $(\approx 20 \mathrm{~mm} \times 10 \mathrm{~mm}$ ) were fixed to a metal support and placed downwards inside a liquid cell with quartz windows, full of DD water. Air bubbles $(3-6 \mu \mathrm{L})$ were formed on the material's surface using a micrometric syringe with an inverted needle. Images of the bubbles were acquired at predefined times, during 10 min using a video camera (JAI CV-A50, JAI A/S, Grosswallstadt, Germany) mounted on an optical microscope (M3Z, Wild, Heerbrugg, Switzerland) and connected to a frame grabber (DT3155, Data Translation, Norton, MA, USA). Image analysis was done through the ADSA-P (Axisymmetric Drop Shape Analysis Profile) software. At least five bubbles were done per sample, being used three strips for each case.

\subsubsection{Mechanical Properties}

The materials' mechanical properties under compressive and tensile loads were evaluated using an Instron ${ }^{\circledR} 5566$ Universal Testing machine from Instron Corporation (Norwood, MA, USA) with a load cell of $10 \mathrm{kN}$. Bluehill ${ }^{\circledR} 2$ software was used to acquire the experimental data. All samples were tested in the hydrated state at room temperature.

Compression tests were done in unconfined mode. The samples were compressed to $70 \%$ strain, at a strain rate of $0.1 \mathrm{~mm} \cdot \mathrm{s}^{-1}$. A preload of $2 \mathrm{~N}$ was applied before each test. For each experimental condition, six discs (diameter $10 \mathrm{~mm}$ ) were utilized. The compressive elastic modulus, strength, and toughness were determined from the acquired engineering stress-strain curves.

Tensile tests were performed with dumbbell-shaped specimens of $2.5 \mathrm{~mm}$ width (overall width $8 \mathrm{~mm}$ ) and $10 \mathrm{~mm}$ gauge length (total length $30 \mathrm{~mm}$ ). The specimens were pulled at an extension rate of $0.5 \mathrm{~mm} \cdot \mathrm{s}^{-1}$ until failure. Measurements were made on at least four replicates of each hydrogel type. The elastic modulus, elongation to break, ultimate tensile strength, and toughness were calculated from the obtained engineering stress-strain curves.

\subsubsection{Tribological Behavior}

Tribological performance of hydrogels against 316L stainless steel (SS) balls (diameter $6 \mathrm{~mm}$, Luis Aparicio SL, Barcelona, Spain), was assessed in reciprocal oscillating mode, using a ball-on-disc geometry configuration in a TRM 1000 (Wazau, Berlin, Germany) tribometer. Discs of hydrated samples (diameter $75 \mathrm{~mm}$ ) were pre-equilibrated for $24 \mathrm{~h}$ in the lubricating media, PBS (Sigma-Aldrich, Saint Louis, MO, USA) solution, before testing. The experiments were performed at room temperature over a stroke length of $10 \mathrm{~mm}$, with a tangential sliding velocity of $43 \mathrm{~mm} \cdot \mathrm{s}^{-1}$ and a sliding distance of $13 \mathrm{~m}$. The normal contact loads applied varied between 20 and $60 \mathrm{~N}$, which correspond to maximum Hertzian contact pressures in the range of 10 to $17 \mathrm{MPa}$ and mean compressive pressures of 7 to $11 \mathrm{MPa}$. All measurements were done at least in triplicate for each type of sample and testing condition. Wear mechanisms in the best performing system were identified through SEM analysis, carried out as described in Section 2.2.1. 


\subsubsection{Drug Loading and Release}

For the drug loading/release assays, A150 samples (diameter $8 \mathrm{~mm}$ ) were dried at $37^{\circ} \mathrm{C}$ for four days and their weight was measured for further calculations. Loading was carried out by direct soaking $\left(3\right.$ days, $37^{\circ} \mathrm{C}$ ) of the discs in diclofenac sodium salt (DFN, $\geq 98 \%$, Sigma-Aldrich, Saint Louis, MO, USA) or ketorolac tromethamine (KTL, $\geq 99 \%$, Sigma-Aldrich, Saint Louis, MO, USA) solutions $\left(2 \mathrm{mg} \cdot \mathrm{mL}^{-1}\right)$ prepared in PBS (Sigma-Aldrich, Saint Louis, MO, USA), or by soaking them in the drug solutions after preloading with DL- $\alpha$-Tocopherol (Vit.E, $>97 \%$, Alfa Aesar ${ }^{\mathrm{TM}}$, Thermo Fisher Scientific, Kandel, Germany) or Vit.E + cetalkonium chloride (CKC, Sigma-Aldrich, Saint Louis, MO, USA). In this case, discs were incubated ( $3 \mathrm{~h}$, room temperature) in Vit.E $(4 \% \mathrm{~m} / \mathrm{v})$ or Vit.E $(4 \% \mathrm{~m} / \mathrm{v})+$ CKC $(0.1 \% \mathrm{~m} / \mathrm{v})$ solutions prepared in ethanol $\left(96 \% \mathrm{v} / \mathrm{v}\right.$, Manuel Vieira \& $\mathrm{C}^{\mathrm{a}}$, Torres Novas, Portugal), using a ratio of $0.15 \mathrm{~mL}$ of solution $/ \mathrm{mg}$ of dry sample. Then, samples were blotted with absorbent paper, dried overnight at $37^{\circ} \mathrm{C}$ and weighted. The increase in the weight of Vit.E and Vit.E + CKC pre-loaded samples was $1.5 \pm 0.5 \%$ and $1.8 \pm 0.5 \%$, respectively.

After, loaded discs were removed from the drug solutions, rinsed with DD water, blotted with absorbent paper and immersed in $3 \mathrm{~mL}$ of PBS solution to perform the drug release experiments. The samples were kept at $37^{\circ} \mathrm{C}$ and $180 \mathrm{rpm}$ in a shaker (Incubating Mini Shaker, VWR International, Alfragide, Portugal) and, at predetermined times, aliquots of $0.3 \mathrm{~mL}$ of the supernatant solution were collected and replaced with the same volume of fresh PBS until the release curve plateau was reached. The amount of drug released was determined by measuring the absorbance of the collected solutions at $276 \mathrm{~nm}$ for DFN, and $315 \mathrm{~nm}$ for KTL, with a UV-Vis spectrophotometer (Multiskan ${ }^{\mathrm{TM}}$ GO Microplate Spectrophotometer, Thermo Fisher Scientific, Kandel, Germany). Calibration curves for each drug were obtained with solutions of different concentrations. Each experimental condition was tested at least in triplicate.

\subsubsection{HET-CAM Test}

Hen's Egg Test on the Chorioallantoic Membrane (HET-CAM) was carried out using fertilized chicken eggs $(\approx 60 \mathrm{~g}$ ) provided by Sociedade Agrícola da Quinta da Freiria (Bombarral, Portugal), following the ICCVAM recommended test [44]. The eggs were incubated at $37^{\circ} \mathrm{C}$ and $60 \%$ of relative humidity for nine days, being rotated $180^{\circ}$ three times/day. On the last day of incubation, the eggs were placed with the wider extreme upward and a circular cut (diameter $\approx 20 \mathrm{~mm}$ ) was made at the air chamber zone to remove the eggshell, using a Dremel ${ }^{\circledR} 3000$ rotary tool (Dremel Europe, Breda, Netherlands). The inner membrane was hydrated with $1.5-2 \mathrm{~mL}$ of sodium chloride ( $\mathrm{NaCl}$, $\geq 99 \%$, PanReac AppliChem, Darmstadt, Germany) aqueous solution at $0.9 \%$ and the eggs were returned to the incubator for $30 \mathrm{~min}$. Then, the saline solution was removed, and the inner membrane was carefully taken off with tongs, to expose the CAM. Any defective egg was discarded.

Hydrated A150 discs (diameter $8 \mathrm{~mm}$ ), non-loaded or loaded with DFN or KTL (both alone or together with Vit.E + CKC) in the same conditions of the ones prepared for the drug loading/release experiments, were rinsed with DD water, blotted, and directly placed on the eggs' CAM. Aliquots $(300 \mu \mathrm{L})$ of aqueous solutions of $\mathrm{NaCl}$ at $0.9 \%$ and sodium hydroxide ( $\geq 99 \%$, Merck, Darmstadt, Germany) at $0.1 \mathrm{M}$, poured on the eggs' CAM were used as negative and positive controls, respectively. The CAMs were observed for $5 \mathrm{~min}$, looking for any signs of vascular lysis, hemorrhage, or coagulation. At least three eggs were used for each experimental condition.

\subsubsection{Cytotoxicity Evaluation}

A150 discs (diameter $5 \mathrm{~mm}$ ) non-loaded or loaded with DFN or KTL (both alone or together with Vit.E + CKC) were dried for four days at $37^{\circ} \mathrm{C}$ and sterilized with $\gamma$-radiation using a dose of $25 \mathrm{kGy}$, which is known to ensure the "overkill" of microorganisms. The radiation was applied at a rate of $12.4 \mathrm{kGy} \cdot \mathrm{h}$ using a ${ }^{60} \mathrm{C}$ source. Immediately prior to cytotoxicity evaluation, samples were aseptically rehydrated for $1 \mathrm{~h}$ in PBS solution (A150), and DFN or KTL solutions (A150 [DFN] and A150 [DFN 
+ Vit.E + CKC] or A150 [KTL] and A150 [KTL + Vit.E + CKC], respectively) prepared in PBS with a drug concentration of $2 \mathrm{mg} \cdot \mathrm{mL}^{-1}$. These solutions were also sterilized before used by steam heat $(20 \mathrm{~min}$, $121^{\circ} \mathrm{C}$ and 1 bar) using a vertical steam sterilizer Uniclave 88/75L (A.J. Costa, Cacém, Portugal), as it is known that DFN and KTL suffer degradation when exposed to $\gamma$-radiation in solution [42,45].

The in vitro cytotoxicity test was carried out by indirect contact according to ISO 10993-5:2009 [46]. NIH/3T3 (Swiss mouse embryo) fibroblasts (93061524, Sigma-Aldrich, Saint Louis, MO, USA) were sub-cultured in Dulbecco's Modified Eagle's Medium (DMEM, Sigma-Aldrich, Saint Louis, MO, USA) supplemented with $10 \%$ of fetal bovine serum (FBS, Sigma-Aldrich, Saint Louis, MO, USA) and $1 \%$ penicillin-streptomycin solution $\left(10,000 \mathrm{U} \cdot \mathrm{mL}^{-1}\right.$ of penicillin and $10 \mathrm{mg} \cdot \mathrm{mL}^{-1}$ of streptomycin, Sigma-Aldrich, Saint Louis, MO, USA) using T75 flasks with filter caps (Thermo Fisher Scientific, Kandel, Germany). The cells were grown in a humidified incubator at $37{ }^{\circ} \mathrm{C}$ in air containing $5 \%$ $\mathrm{CO}_{2}$, as described elsewhere [47]. Morphological examinations of cells were performed daily using an inverted light microscope (Axiovert ${ }^{\circledR}$ 25, ZEISS Microscopy, Jena, Germany). Only cells with less than ten passages were utilized to minimize differences in cell cycle and genetic background.

The NIH/3T3 cells were seeded into 24 -well plates at a density of $1 \times 10^{5}$ cells/well, and incubated for $24 \mathrm{~h}$ at $37{ }^{\circ} \mathrm{C}$ under a humidified atmosphere containing $5 \% \mathrm{CO}_{2}$ in air, to allow cells to reach $80 \%$ confluence before exposure to the samples. After that, the culture medium was replaced by the same quantity of fresh DMEM $600 \mu \mathrm{L} /$ well) and the cell culture inserts (Corning ${ }^{\circledR}$ Transwell ${ }^{\circledR}$ permeable supports with polycarbonate membrane $[\varnothing=6.5 \mathrm{~mm}$, pore size $=8.0 \mu \mathrm{m}]$, VWR International, Alfragide, Portugal), were placed in the wells. The samples were carefully implanted into the inserts, and an additional $100 \mu \mathrm{L}$ of DMEM/well was added on the top of the samples to ensure that the materials were completely immersed into the culture medium. As negative control, cells were treated with DMEM, and as positive control cells were incubated with DMEM containing 7.5\% v/v of dimethyl sulfoxide (DMSO, $\geq 99.9 \%$, Sigma-Aldrich, Saint Louis, MO, USA). After incubation for $24 \mathrm{~h}$ at $37{ }^{\circ} \mathrm{C}$ under a humidified atmosphere with $5 \% \mathrm{CO}_{2}$, the inserts containing the samples and the culture medium were both removed from each well, and $300 \mu \mathrm{L} /$ well of a 3-(4,5-dimethyl-2-thiazolyl)-2,5-diphenyl-2H-tetrazolium bromide (MTT, Sigma Aldrich, Saint Louis, MO, USA) solution at $0.5 \mathrm{mg} \cdot \mathrm{mL}^{-1}$ prepared in a serum-free DMEM, was added to the cells. After $3 \mathrm{~h}$ of incubation in the same conditions, $450 \mu \mathrm{L} /$ well of MTT solvent, consisting of octylphenoxy poly(ethyleneoxy)ethanol (IGEPAL ${ }^{\circledR}$ CA-630, Merck, Darmstadt, Germany) at $0.1 \%$ prepared in isopropanol (Sigma-Aldrich, Saint Louis, MO, USA) with hydrochloric acid (Sigma-Aldrich, Saint Louis, MO, USA) at $4 \mathrm{mM}$, was added to dissolve the formazan crystals formed during the reaction time. The absorbance was measured at $595 \mathrm{~nm}$ using a microplate reader AMP Platos R 496 (AMEDA, Labordiagnostik, Graz, Austria). The percentage of cell viability was acquired by comparison of the absorbances obtained for each experimental condition with the negative control, considered with $100 \%$ cell viability. All the experiments were done at least in quadruplicate.

\subsubsection{Statistical Analysis}

Statistical analysis was performed with IBM ${ }^{\circledR}$ SPSS ${ }^{\circledR}$ Statistics (v.25) software (IBM Corporation, Armonk, NY, USA). Quantitative data were expressed as mean \pm standard deviation. Shapiro-Wilk test was used to check the normality of the results' distributions. When this was verified, the similarity of variances was evaluated using the Levene's test. If variances between groups were similar, One-Way ANOVA was applied, followed by a Tukey HSD test for multiple comparisons, as needed. When the equality of variances assumption was not met, the data were analysed by Welch's ANOVA and Dunnett T3 test to identify significant different pairs of groups. In cases where normality was not verified, Kruskal-Wallis tests followed by Mann-Whitney U testes adjusted using the Bonferroni correction, were applied for pairwise comparison between groups, as required. The significance level was set at $p \leq 0.05$. The statistically significant differences between groups were indicated by asterisks. 


\section{Results and Discussion}

\subsection{Morphology}

Figure 1 exhibits the surface and cross-section images of the different samples, as observed by scanning electron microscopy (SEM). All hydrogels give rise to transparent and homogeneous films, and almost all reveal non-porous dense structures with some wrinkles, with no evident effect regarding the variations in processing conditions. This indicates that the first processing step essentially determines the microstructure of the samples. The only exception is with HHP gel, which presents several tiny pores in the cross-sectional images, but not on its surface. This morphological change is possibly due to the breakage of some connections during pressurization treatment.

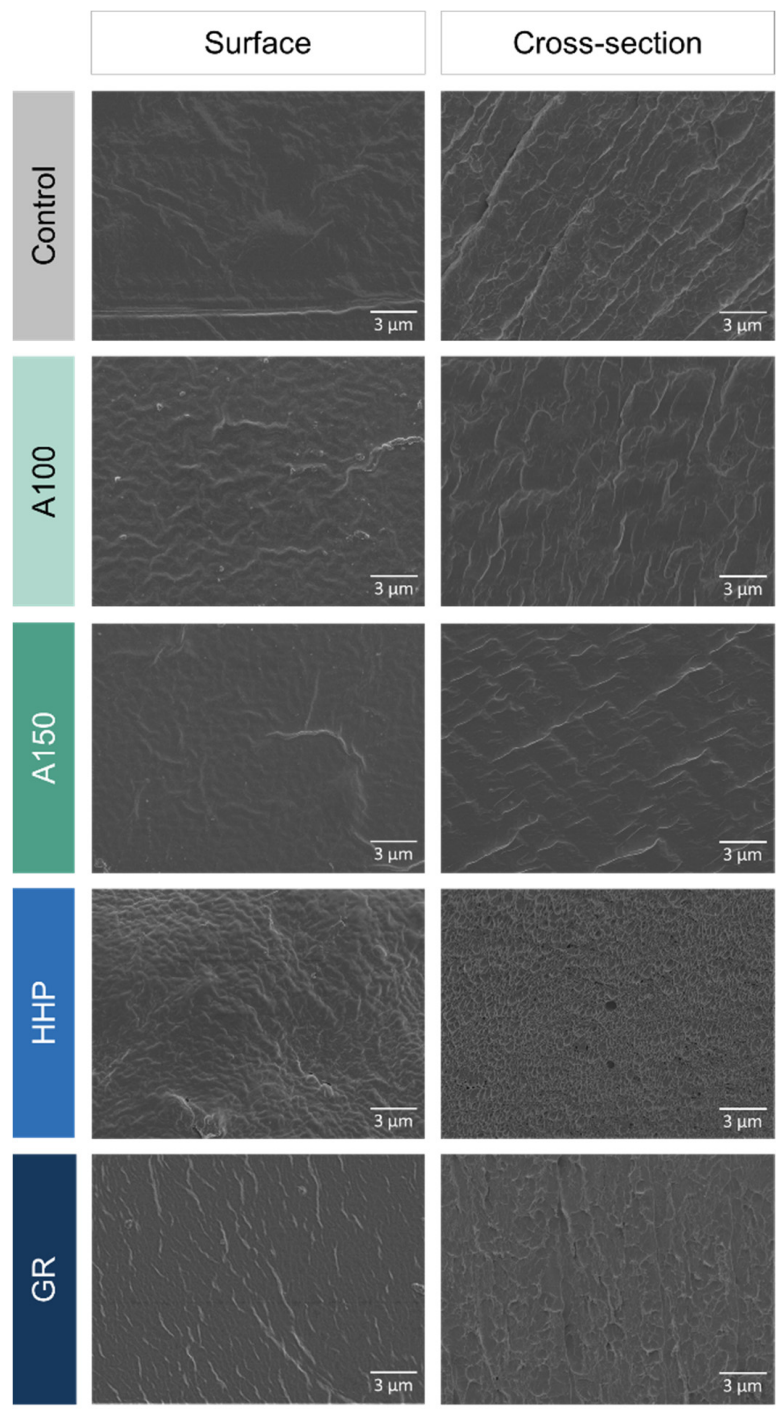

Figure 1. SEM images of the surface and cross-section of PVA samples.

\subsection{Water Uptake}

PVA is a hydrophilic polymer, which contains in its structure a large number of hydroxyl groups than can form hydrogen bonds with free water molecules. To evaluate the effect of different processing treatments on the water absorption capacity of PVA hydrogels, the equilibrium water content (EWC) values were determined. As shown in Figure 2, Control and A100 samples have comparable water contents $(p=0.575)$, about $50 \%$, which drops to $\approx 40 \%$ in samples annealed at $150{ }^{\circ} \mathrm{C}(\mathrm{A} 150)$. This can be attributed to the additional crosslinking of the polymeric matrix induced by heat, which increases its 
crystallinity and tightens the materials' network [26,27]. Gonzalez et al. [28] observed similar behavior with PVA FT hydrogels: the increase in annealing temperature from $100{ }^{\circ} \mathrm{C}$ to $140{ }^{\circ} \mathrm{C}$ led to a significant reduction in the swelling degree of the material. Exposure to $75 \mathrm{kGy}$ of $\gamma$-radiation also decreased the EWC to values in the order of $40 \%$. Other authors reported on the reduction of the swelling ratio with the increase of radiation dose in PVA-based hydrogels, attributing this phenomenon to the promotion of network crosslinking by radiation action [48,49]. Regarding HHP treatment, there was an opposite effect, significantly increasing the water content in the hydrogel, reaching around $80 \%$. HHP is widely used as a post-processing method in the food industry for destroying pathogenic microorganisms, and therefore, a large number of scientific works have focused on studying its effect on biomolecules (e.g., proteins), demonstrating that it leads to changes in the secondary, tertiary, and quaternary structure of the molecules, due to the rupture of the physical bonds [50]. However, there are no investigations focusing on the effect of HHP on crosslinked hydrogels. The observed increase for the EWC suggests that it may induce the destruction of the hydrogen bonds between the polymeric molecules of PVA, causing an increase in the chain mobility and facilitating the network expansion with the absorption of water.

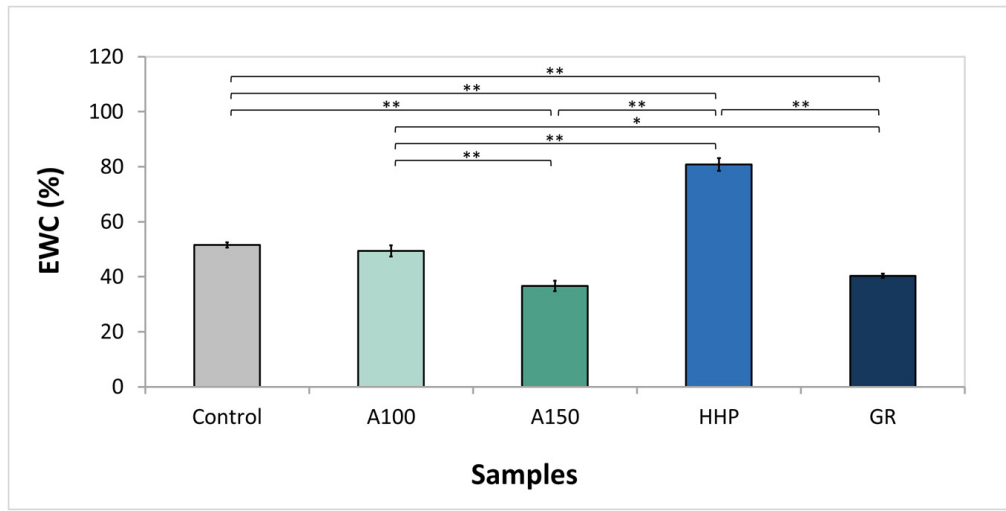

Figure 2. Equilibrium water content of PVA samples. Data tested by ANOVA $(p<0.0001)$ followed by Tukey HSD test. Statistical significance: ${ }^{*} p<0.001,{ }^{* *} p<0.0001$.

\subsection{Wettability}

The surface wettability is an important characteristic, as it will influence the interaction of an implanted material with the surrounding biological fluid and tissue molecules, determining the body's response to it. Figure 3 displays the values of water contact angles obtained for the different types of hydrogels. All of them are hydrophilic, with water contact angles $\leq 37^{\circ}$. Post-processing treatments led to a minor reduction in the contact angle, and thus a negligible increase in hydrophilicity. However, there was no evidence of a statistical difference between all the samples $(p=0.06)$.

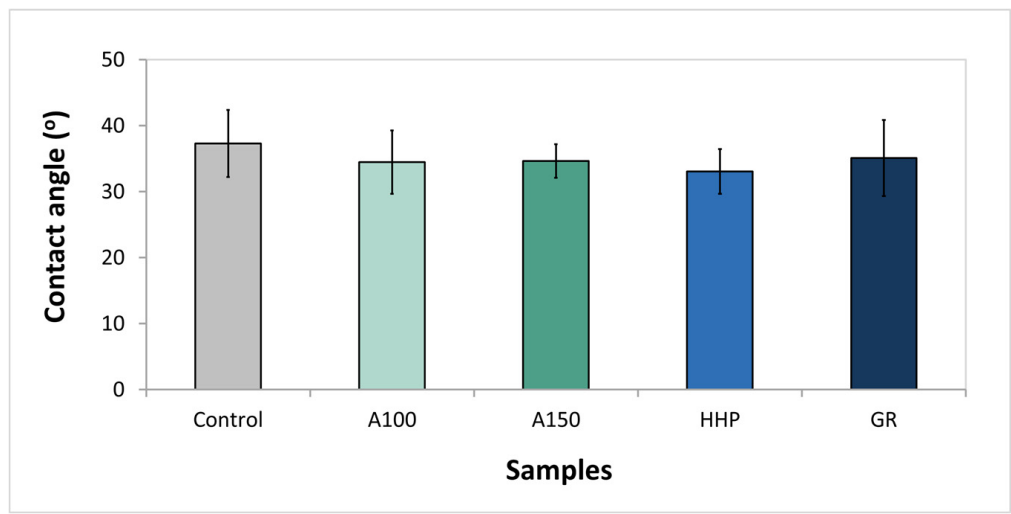

Figure 3. Water contact angles with PVA samples. Data analysed by Kruskal-Wallis $(p>0.05)$. 


\subsection{Mechanical Properties}

Human joints are subjected to compression and tensile stresses, and so, it is crucial to evaluate the mechanical response of the cartilage substitute materials to such efforts.

Figure 4a shows typical compressive stress-strain curves obtained for the different materials. A non-linear behavior can be observed in all cases. Annealing at $100{ }^{\circ} \mathrm{C}$ and exposure to $\gamma$-radiation did not induce major changes in the mechanical response of the hydrogels to the compression load. However, annealing at $150{ }^{\circ} \mathrm{C}$ and HHP had visible opposite effects: for a given common strain value, a higher stress was required on the A150 samples and a much lower one on the HHP gels. The elastic modulus, compressive strength, and toughness (area under the curves) were calculated for each case and are given in Figure $4 \mathrm{~b}-\mathrm{d}$, respectively. The modulus of elasticity of all samples increases with the strain (Figure $4 \mathrm{~b}$ ). Nevertheless, in absolute terms, its variation is much lower in HHP gels. Indeed, HHP materials are less rigid when compared to all others, with a maximum modulus of elasticity of $5 \pm 3 \mathrm{MPa}(p<0.05)$. These hydrogels also present the lowest maximum strength $(4 \pm 2 \mathrm{MPa})$ and toughness $\left(0.6 \pm 0.2 \mathrm{MJ} \cdot \mathrm{m}^{3}\right)$. A150 samples demonstrate the highest resistance at compression, being more rigid, and reaching values of $35 \pm 8 \mathrm{MPa}$ for compressive strength and $8 \pm 2 \mathrm{MJ} \cdot \mathrm{m}^{3}$ for toughness. GR and A100 samples have an intermediate performance, not very different from the untreated samples.
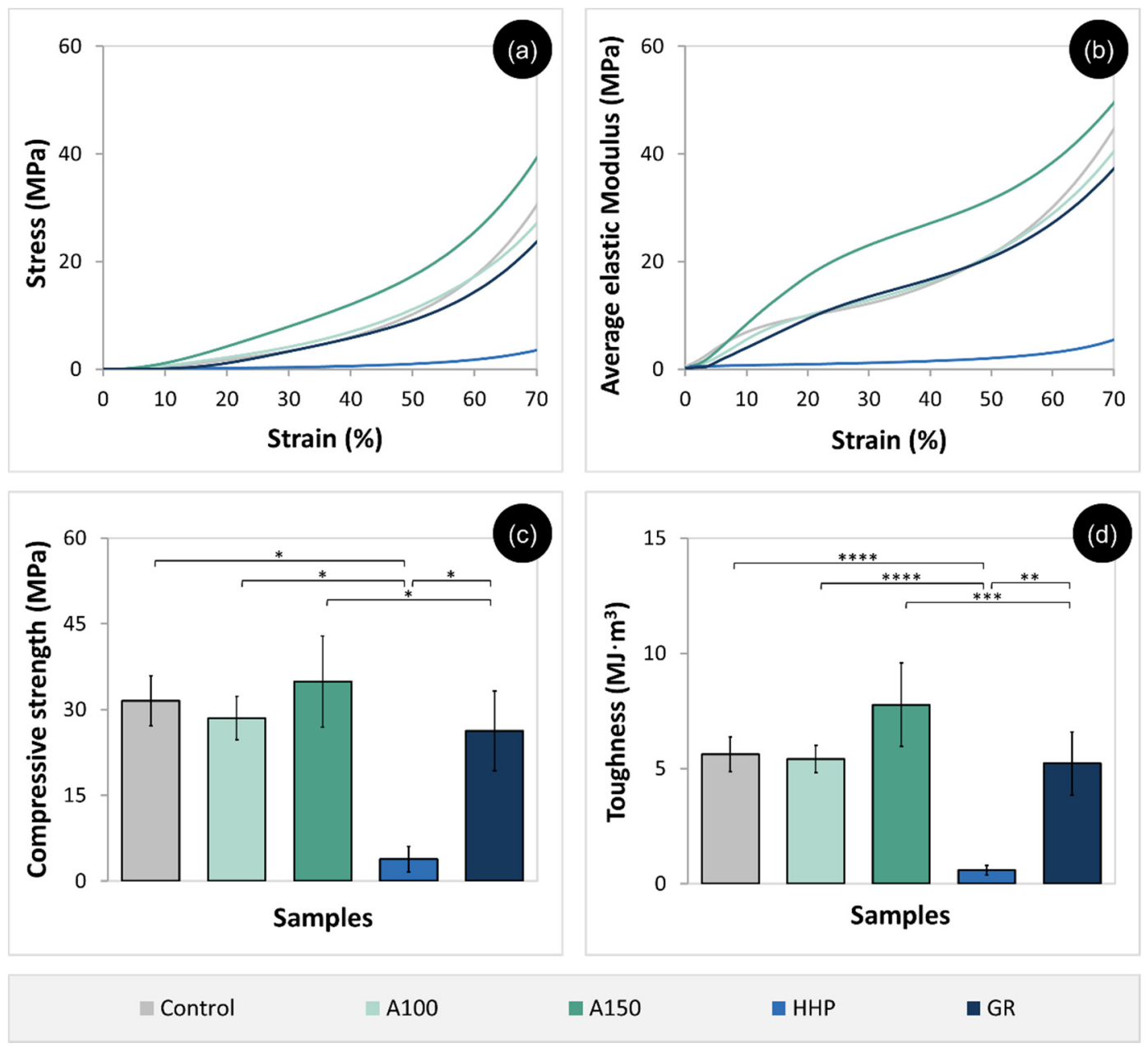

Figure 4. Typical compression stress-strain curves (a), elastic modulus (b), compressive strength (c), and toughness (d) of PVA samples. For the maximum values of the average elastic Modulus and for the compressive strength, data were analysed by Kruskal-Wallis $(p<0.005)$ followed by Mann-Whitney U with Bonferroni adjustment. For the toughness, data were tested by Welch's ANOVA $(p<0.0001)$ followed by Dunnett T3 test. Statistical significance: ${ }^{*} p<0.05,{ }^{* *} p<0.005,{ }^{* * *} p<0.0005,{ }^{* * * *} p<0.0001$. 
Concerning tensile behavior, representative stress-strain curves of all tested materials are provided in Figure 5, together with their elastic modulus, elongation to break (maximum strain), ultimate tensile strength (maximum stress), and toughness. As with compression, hydrogels exhibit a nonlinear behavior in tensile (Figure 5a). However, their elastic modulus decreases with the increasing strain (Figure 5b). The mechanical properties of the HHP samples are worsened, with a strong decrease in the modulus of elasticity (which comes to values below $1 \mathrm{MPa}$ ), elongation to break (196 $\pm 35 \%)$, ultimate tensile strength $(0.9 \pm 0.1 \mathrm{MPa})$ and toughness $\left(1.0 \pm 0.4 \mathrm{MJ} \cdot \mathrm{m}^{3}\right)$. The remaining treatments improved the tensile properties of gels, but annealing at $150{ }^{\circ} \mathrm{C}$ led to the most notable differences: it increased the elastic modulus to a value that reaches $22 \pm 2 \mathrm{MPa}$ at lower strain values $(p<0.005)$, and approximately doubled the tensile strength $(18 \pm 1 \mathrm{MPa})$ and toughness $\left(43 \pm 5 \mathrm{MJ} \cdot \mathrm{m}^{3}\right)$; the maximum elongation remained similar to that observed for the untreated samples and was higher than $400 \%$.
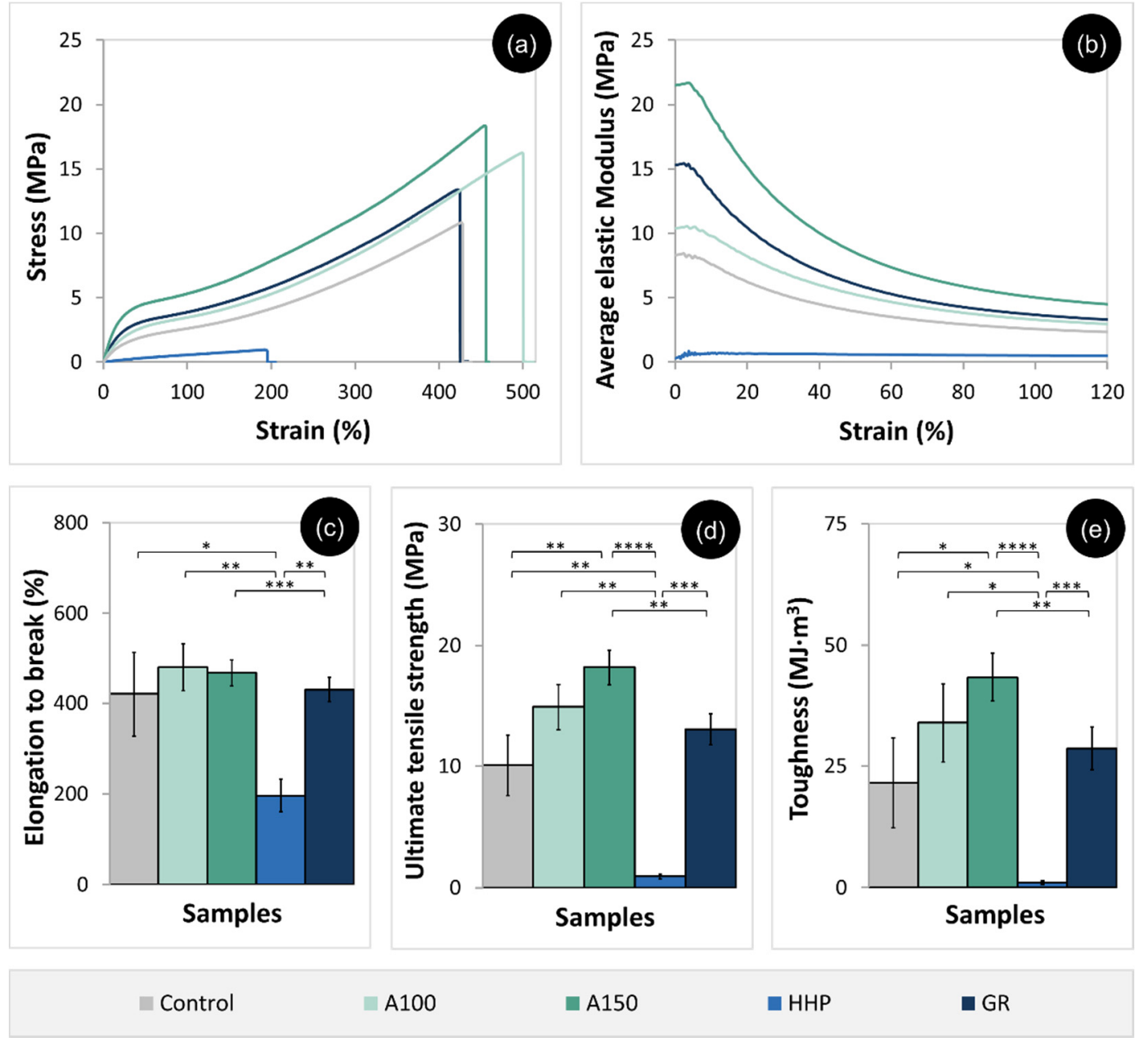

Figure 5. Typical tensile stress-strain curves (a), elastic modulus (b), elongation to break (c), ultimate tensile strength (d), and toughness (e) of PVA samples. Data were evaluated by Welch's ANOVA $(p<0.0001)$ followed by Dunnett T3 test. Statistical significance: ${ }^{*} p<0.05,{ }^{* *} p<0.005,{ }^{* * *} p<0.0005$, $* * * * \quad p<0.0001$.

From the analysis of the results, it is clear the distinct behavior of the variation of the elastic modulus of the materials with the strain under compression or tensile loads. As noted earlier, the modulus of elasticity increases with the strain under compression, while in tensile, it goes down till reaching an almost constant value. Few authors presented stress-strain curves of hydrogels in the two situations (e.g., [51,52]). Li et al. [51], obtained comparable S-S curves with acrylamide hydrogels and related the 
materials' behavior to the entanglement between polymer chains in the hydrogel network, stating that the slippage or rearrangement of entangled polymer chains is responsible for the energy dissipation when hydrogels are subjected to compressive or tensile forces. That is, compression enhances the interpenetration of polymer chains and release of water contained in the hydrogel, hardening the material - a phenomenon that also occurs with natural cartilage, preventing the exudation of more fluid above certain loads, and is considered a tissue protection mechanism [53]. On the other hand, during the initial phase of stretching, a linear deformation occurs with a high modulus of elasticity due to the entropic elasticity of the network [54]. The high initial modulus depends mainly on the density of intermolecular hydrogen bonds. As stress rises, these bonds, which are largely responsible for the physical crosslinking of the material, can break and recombine dynamically, leading to the homogenization of the gel network [55] and a lower and practically constant modulus of elasticity. Therefore, the results of the mechanical tests, both in compression and tensile, agree with what was referred in the previous sections and confirm that the annealing at $150{ }^{\circ} \mathrm{C}$ is the treatment that leads to the highest degree of crosslinking of the hydrogel. Fukumori et al. [27] came to the same conclusion. According to these authors, small crystallites act as crosslinking points, and annealing promotes the formation of larger crystallites improving the mechanical resistance of the materials. In turn, HHP had a deleterious effect on the mechanical properties of the gel, which should be related to the break of hydrogen bonds between polymer chains of PVA during the high-pressure treatment, as stated in Section 3.2. With regard to $\gamma$-radiation, although no significant differences were observed in the compressive behavior of irradiated gels compared to that of the Control samples, a slight improvement was seen when these samples were under tensile efforts. Shi et al. [52] studied the effect of $\gamma$-radiation (doses between 50 and $150 \mathrm{kGy}$ ) on the mechanical properties of FT hydrogels made of PVA/polyvinyl pyrrolidone and found that they depended significantly on the applied dose. Hydrogels treated with $100 \mathrm{kGy}$ achieved the best mechanical properties. The authors explained that, for moderate doses, irradiation leads to the formation of polymer radicals due to the abstract of hydrogen atoms from the $-\mathrm{CH}(\mathrm{OH})-$ and/or $-\mathrm{CH}_{2}-$ groups of PVA, that interact among them, promoting the formation of hydrogen bonds and crosslinking in the materials, and hence, improving their mechanical resistance and elastic modulus. However, at higher doses, crosslinking of polymers reaches to saturation level, were the keto-enol tautomerization of $\alpha$-PVA radicals prevails and PVA chains may undergo scission, decreasing mechanical strength and hydrogel modulus [55].

The data found in the literature for the mechanical properties of natural cartilage present a large dispersion, owing to differences in test conditions, the origin of the tissue (human vs. animal), location, and type (e.g., hyaline and fibrocartilage). Regarding the compressive behavior, values between 25 and $65 \mathrm{MPa}$ are reported for the compressive strength, while elastic modulus ranges from 0.13 to $1.91 \mathrm{MPa}$ [53]. The compressive strength of A150 samples falls in that interval. But the modulus of elasticity is difficult to compare, as it depends on the material's strain. However, it increases with the compression ratio, similar to what occurs with cartilage [56]. Such characteristic is very important when considering the material for replacing the tissue. Tensile tests of cartilage show values of modulus of elasticity varying from 0.5 to $30 \mathrm{MPa}$ and ultimate tensile strength ranging from 2 to $50 \mathrm{MPa}$. The corresponding values obtained for samples A150 are within these ranges. No toughness values were found for cartilaginous tissue for comparison.

\subsection{Tribological Behavior}

Figure 6 provides the average CoF values for samples tested against 316L SS in PBS solution, under various loads (between 20 and $60 \mathrm{~N}$ ). For each type of sample, a $10 \mathrm{~N}$ incremental rise of the applied load was done, whenever the material resisted to the previous load.

The HHP samples did not withstand the initially used pressure $(20 \mathrm{~N})$, and for this reason, no values of CoF were measured. The failed attempt to assess the frictional properties of the HHP gels may be explained by its lower resistance to compression loads (see Section 3.4). The breakage of 
some physical crosslinking connections should cause a decrease in the superficial shear strength and an increase in the adhesion of the counterbody.

As seen from the graph, GR samples only withstand loads up to $30 \mathrm{~N}$ without being destroyed. This means that although no significant changes had been observed in the compressive behavior of the irradiated hydrogels, they became less resistant to wear. Some degradation may have occurred, as it is known that irradiation may also induce random degradation in the polymer chains [52].

A global analysis of the results shows that, for each type of sample, as the normal force increases, the friction values also increase. PVA is a very resilient and viscoelastic material which can easily suffer deformation. Higher applied loads lead to a larger contact area between the counterbody and the hydrogel surface, thus to a deeper deformation of the gel, favoring the increase in the sliding friction force, and consequently leading to higher $\mathrm{CoF}$ values. On the other hand, larger loads would enhance the release of water from hydrogels to the friction interface, which would benefit lubrication, contributing to the reduction of CoF. The obtained results show that the first effect should prevail. Pan et al. [57,58] studied the frictional properties of a FT PVA gel against titanium alloy and stainless steel balls and came to the same conclusions about the influence of the load.

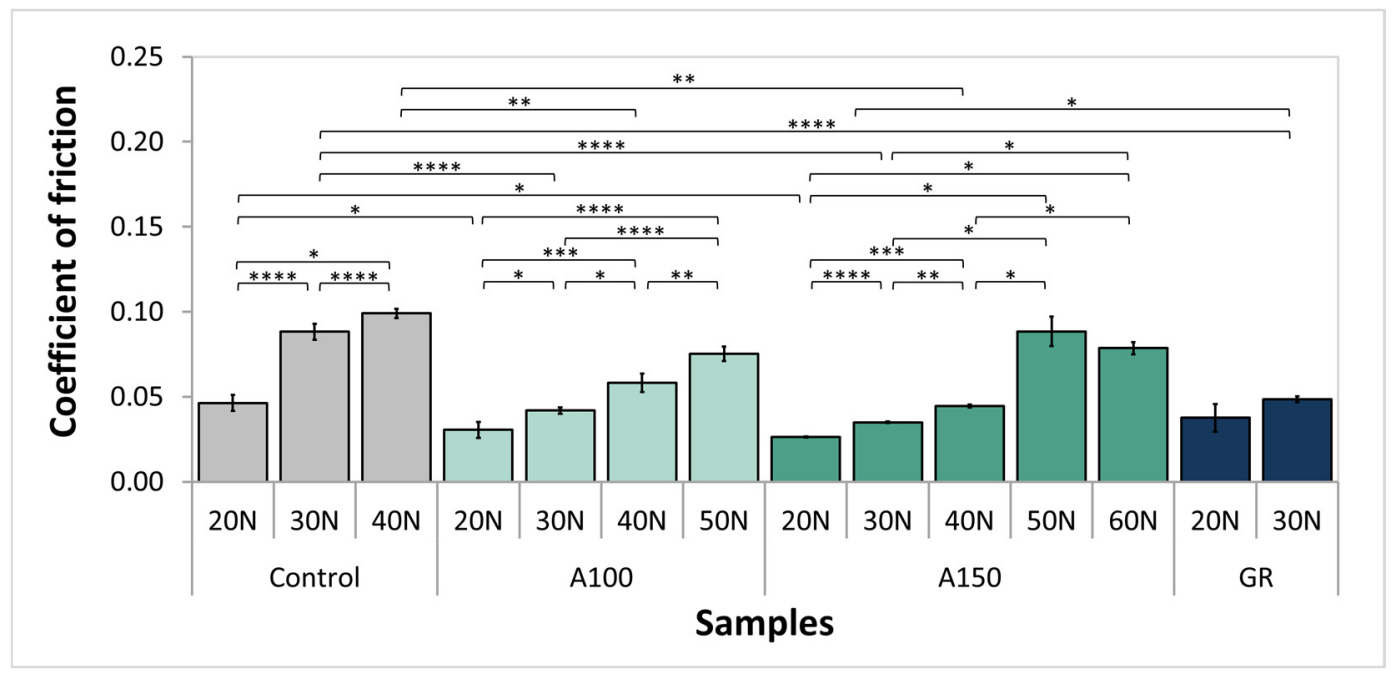

Figure 6. Friction coefficients of PVA samples measured against SS 316L in PBS solution. Comparisons involving Control $(40 \mathrm{~N})$, A100 $(40 \mathrm{~N})$ and A150 (20-60 N) were given by Welch's ANOVA $(p<0.0005)$ followed by Dunnett T3 test. All other comparations were performed by ANOVA $(p<0.01)$ followed by Tukey HSD test. Statistical significance: ${ }^{*} p<0.05,{ }^{* *} p<0.005,{ }^{* * *} p<0.0005,{ }^{* * * *} p<0.0001$.

Another evidence from the graph is that for each tested load, the friction of treated hydrogels is lower than that of Control samples. As indicated above, both annealing and $\gamma$-radiation led to further crosslinking of the gels and improved their crystallinity. This effect limits the activity of the crosslinking points in the chains and impairs conformational changes of chain segments, explaining not only the greater difficulty of water molecules penetrating into the gel matrix [18], which results in smaller EWC values but also to the lower adhesion to the opponent materials, resulting in less friction.

As a final remark, it shall be stressed that the A150 samples exhibit the best frictional behavior, with CoFs ranging from $0.026 \pm 0.002$ to $0.09 \pm 0.01$. For the lowest normal loads $(20-40 \mathrm{~N})$, these hydrogels had the lowest $\mathrm{CoF}$ values. More, they were the only ones that withstand the highest applied normal load $(60 \mathrm{~N})$. Indeed, this behavior was expected based on the superior mechanical performance of these samples, which suffered less deformation and appear to be more resistant to wear.

Friction coefficient values of articular cartilage can vary over a wide range, from as low as 0.002 to as high as 0.6 [8,59-62]. Such variability can be related to a variety of factors, as the geometry of the system, test parameters (e.g., sliding velocity, applied load), and lubricant characteristics. In a recent work [8], we measured the CoF of human cartilage samples against SS in the same testing conditions 
used in this work and obtained the value of $0.19 \pm 0.02$. In agreement, Li et al. [61] reported CoFs between $0.16-0.27$ of human articular cartilage pins against SS plates in Ringer's solution. The friction coefficient values against SS presented in this work were much lower than those found in the literature.

Concerning wear, it was not possible to quantify the wear loss due to experimental limitations. However, the wear mechanisms of the best performing system (A150) were investigated through the analysis of SEM images of the worn surfaces. As mentioned in Section 2.2.5, the normal contact loads applied in the tests $(20-60 \mathrm{~N})$ correspond to maximum Hertzian contact pressures of 10 to $17 \mathrm{MPa}$. The average loads on the human joints depend on their location and do not typically exceed $3 \mathrm{MPa}$, while peak contact stresses rarely reach $10 \mathrm{MPa}$ [63]. Figure 7a depicts the surface of the hydrogel after testing under the most similar conditions of load $(20 \mathrm{~N})$, and no features could be observed. However, with an increased applied load, the wear track is clearly visible (Figure $7 \mathrm{~b}$ ). For example, for $40 \mathrm{~N}$, parallel scratches, indicative of abrasive wear, can be seen. It should be underlined that the tribological tests were carried out under more severe conditions than those found in the human body. In fact, a stainless steel ball was used, which is much harder than the opposing cartilage in a real situation. More, the absence of proteins and other organic molecules in the lubricant media used in the tests, which exist in the pseudosynovial fluid and are known to adsorb on the surfaces, forming a protective layer [64], must also be taken into account.

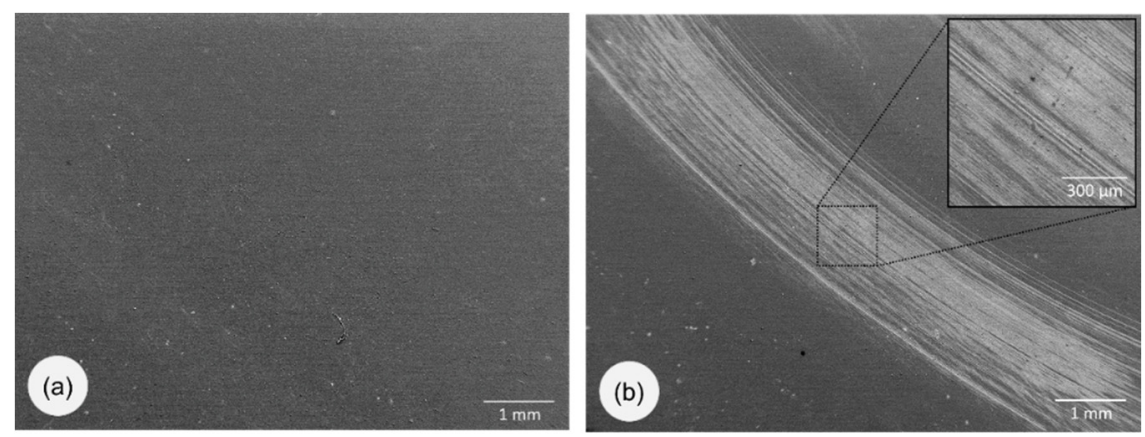

Figure 7. SEM image of the surface of samples A150 after tribological tests against SS 316L in PBS solution, with a load of $20 \mathrm{~N}$ (a) and $40 \mathrm{~N}(\mathbf{b})$. The insert corresponds to a magnification of the wear track.

\subsection{Drug Loading and Release}

A150 samples revealed to be the most resistant and tough and exhibited the best tribological characteristics. For this reason, they were chosen to proceed with the work and were loaded with anti-inflammatories (DFN or KTL). Cumulative release profiles are given in Figure 8 for samples loaded alone with the drugs or together with Vit.E, with and without CKC. The samples loaded only with the drugs have an initial burst on release. This burst effect is usually associated with the diffusion of the free or loosely bound drug from the area near the hydrogel surface into the surrounding medium [65]. Although in both cases, drug release is almost complete within the first $24 \mathrm{~h}$, the amount of DFN loaded/released is nearly double that of KTL. DFN and KTL are both anionic hydrophilic drugs that can bind to the PVA matrix through hydrogen bonds. However, KTL is a larger molecule $(\mathrm{MW} \approx 376 \mathrm{Da})$ and has more places to interact with PVA hydroxyl groups $(\mathrm{OH})$ than DFN $(\mathrm{MW} \approx 318 \mathrm{Da}$ ), which may explain the observed behavior (see the structure of the molecules in Figure S1 in the Supplementary Information). 

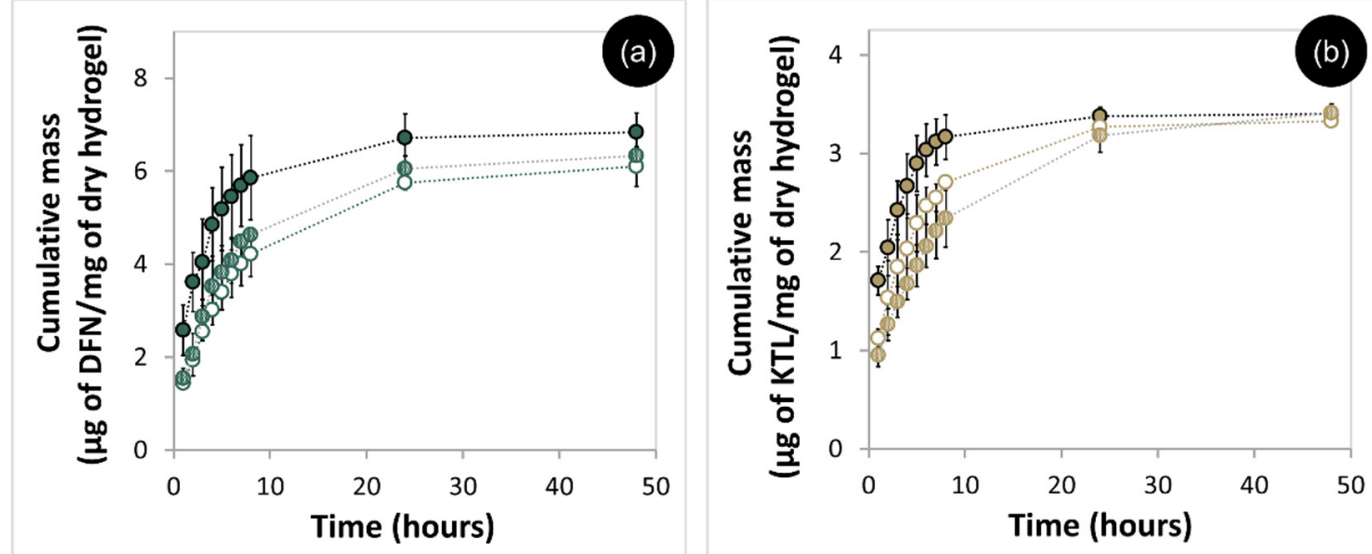

$\begin{array}{ll}0 . A 150[D F N] & \text { O.A150 [DFN + Vit.E] } \\ .0 \cdot A 150[K T L] & . A 150[K T L+\text { Vit.E }]\end{array}$

- A150 [DFN + Vit.E + CKC]

- $A 150[K T L+V i t . E+C K C]$

Figure 8. Cumulative drug release profiles of DFN (a) and KTL (b) from A150 samples, loaded alone, and with Vit.E, with and without CKC. The total amount released of all groups was analysed by ANOVA $(p>0.05)$.

Loading the samples with Vit.E led to a more sustained release of the drugs. Vit.E is a hydrophobic molecule that can form aggregates within the hydrogel matrix, which act as barriers to drug diffusion [40,62]. Peng et al. [66] demonstrated that, due to its negligible solubility in water or PBS, Vit.E is not released from the hydrogels. The extended drug release arises from the long and tortuous path that the drug molecules have to go through inside the polymer matrix. From the analysis of our release profiles, it is evident that the total amount of drug released was not significantly affected by the incorporation of Vit.E $(p>0.05)$, pointing to the existence of a minor interaction between the drugs and the aggregates of Vit.E.

The addition of CKC further improved the release kinetic. This was mainly noticeable for KTL. $\mathrm{CKC}$ is a cationic surfactant that owing to its low toxicity, can be used in biomedical applications to control the release of anionic drugs $[67,68]$. Such drugs interact with high affinity with the surfactant and diffuse along with the material with a diffusivity lower than that of the free drugs, leading to a more prolonged release. Since the pKa of KTL in water (3.5 [69]) is lower than that of DFN (4 [70]), it shall be more dissociated in the aqueous medium, and therefore prone to interact with CKC.

It is not easy to predict the in vivo efficacy of the studied systems. In fact, while in other type of applications it is possible to build mathematical models (e.g., soft contact lenses or intraocular lenses, where the volume and renovation rate of the biological contacting fluids can be estimated) or even perform tests in microfluidic cells that approach the in vivo behavior, in the present case it is difficult to foresee the conditions that the replacement material will face when implanted. Therefore, only in vivo studies would be able to attest the real usefulness of these systems. Even though it is possible to state that the A150 hydrogel loaded with Vit.E + CKC can release the studied drugs in a controlled manner up to $24 \mathrm{~h}$.

\subsection{HET-CAM Test}

The HET-CAM assay of the developing chick embryo was originally validated for toxicity and irritation studies [71,72] and is gaining increasing attention for biomaterials assessment [73,74]. It offers a simple, cost-effective, and short-term in vivo alternative to pre-evaluate biomaterials before animal experimentation $[73,75]$. Besides, the highly vascularized CAM lacks a nervous system, being a less sentient choice for animal testing [74]. Although cartilaginous tissues have an avascular structure, bone and surrounding tissues of the joints are vascularized, thereby creating the need and relevance for performing this test. 
Macroscopic evaluation of the CAM after 5 min exposure to A150 samples before and after loading with DFN and KTL, with or without Vit.E + CKC was done, being the images presented in Figure 9. The irritability evaluation of the systems under study allows us to understand the effect of the material, drugs, and molecules (Vit.E and CKC) used to control the drug release. The results showed that none of the materials caused hemorrhage, lysis, coagulation, or inflammatory response at the time of the study, similarly to what happened with negative control (Ctrl-, $\mathrm{NaCl}$ at $0.9 \%$ ). Therefore, all samples can be qualified as non-irritants. Contrarily, the positive control ( $\mathrm{Ctrl}+, \mathrm{NaOH}$ at $0.1 \mathrm{M})$ led to a severe irritation reaction with almost instant bleeding, blood vessel disintegration, and protein denaturation, which was aggravated during the experiment time.

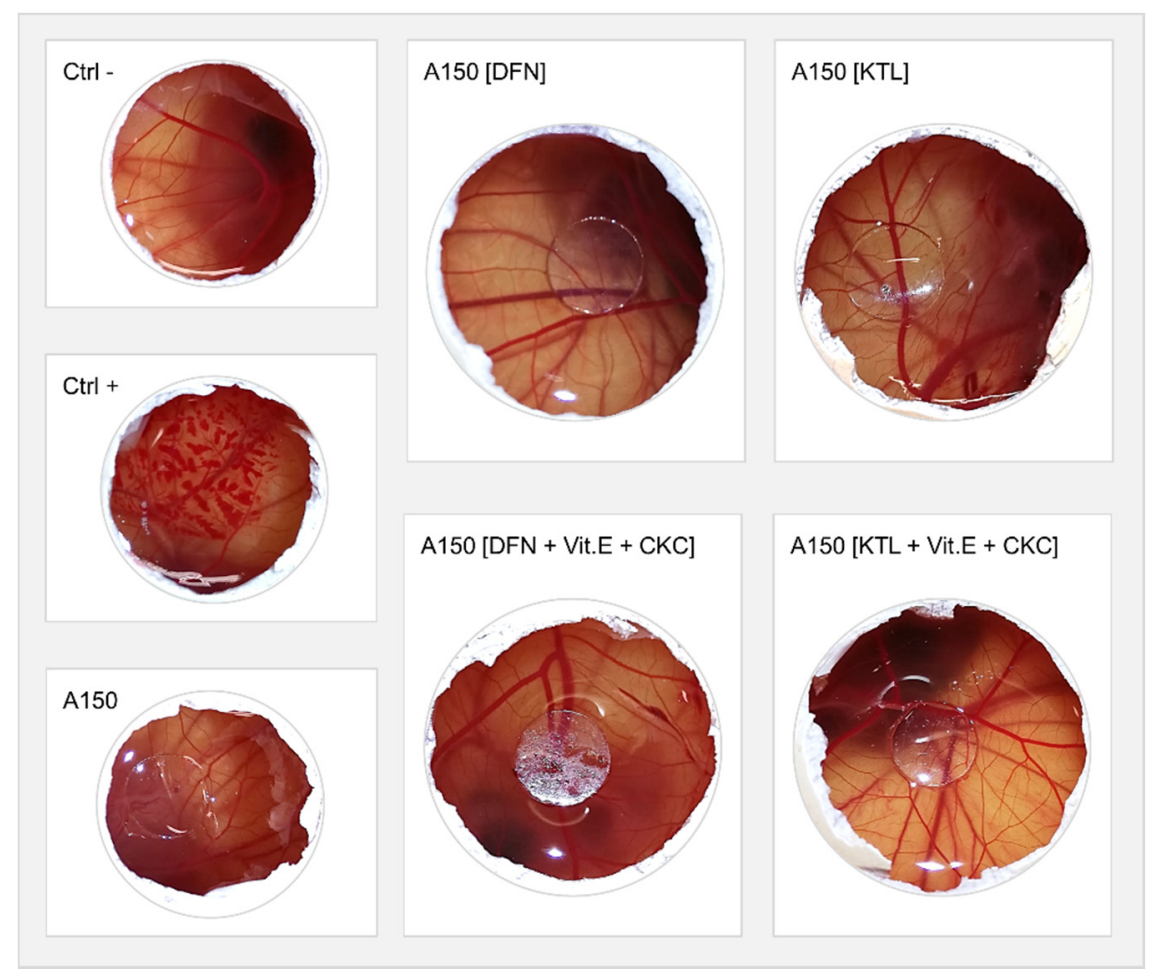

Figure 9. CAM images after incubation for 5 min of A150 samples before and after loading with DFN or KTL, with or without Vit.E + CKC. The negative (Ctrl-) and positive (Ctrl+) controls are also shown.

\subsection{Cytotoxicity Evaluation}

Biomaterials should be biocompatible so as not to harm surrounding tissues when implanted. Numerous authors have demonstrated that PVA hydrogels fulfill this requisite [9]. Incorporation of drugs and other compounds such as Vit.E or CKC into PVA networks may induce cytotoxicity (e.g., due to exceeding toxicity limits or formation of new toxic substances) and compromise the biocompatibility of the materials. For this reason, A150 samples non-loaded or loaded with DFN or KTL, with or without Vit.E + CKC, were evaluated in vitro for cytotoxicity.

The percentage of survival of mouse fibroblast cell line NIH/3T3 after incubation with the control solutions (DMEM and DMEM with DMSO at 7.5\% v/v as Ctrl- and Ctrl+ respectively) and materials are provided in Figure 10a. The morphology of cells incubated with the samples and treated with the controls is also shown in Figure 10b. Incubated cells with all hydrogels were viable up to $24 \mathrm{~h}$ and could proliferate on the culture plates. Cell microphotographs reveal a high number of fibroblasts for Ctrl- and all samples tested, with elongated shapes characteristic of fibroblastic cell morphology, and high cell spread and adhesion, unlike Ctrl+, where a small number of cells is visible. According to ISO 10993-5:2009, a material that allows a cellular viability $\geq 70 \%$ can be considered non-cytotoxic. 
Thus, as all samples show cellular viability close to $100 \%$, it can be concluded that both loaded and non-loaded hydrogels do not possess a cytotoxic effect and can be safely used as biomaterials.
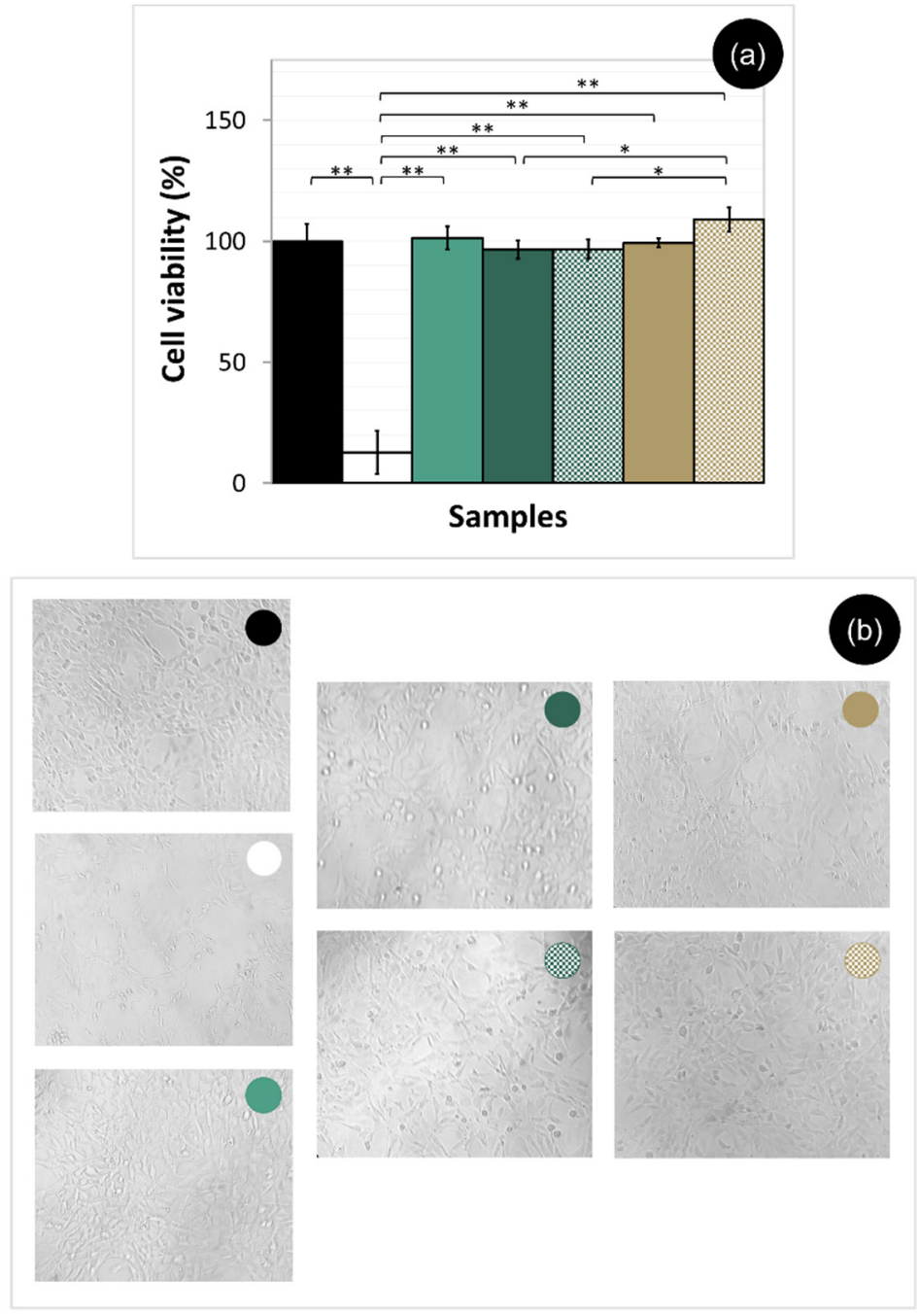

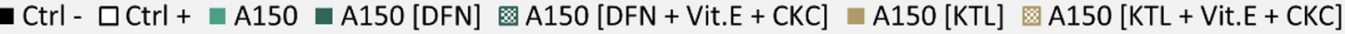

Figure 10. Viability percentage (a) and microscope photographs $(100 \times)(\mathbf{b})$ of NIH/3T3 cells after incubation for $24 \mathrm{~h}$ of A150 samples non-loaded or loaded with DFN or KTL, with or without Vit.E + CKC. The negative (Ctrl-) and positive (Ctrl+) controls are also presented. Data tested by ANOVA $(p<0.0001)$ followed by Tukey HSD test. Statistical significance: ${ }^{*} p<0.05,{ }^{* *} p<0.0001$.

\section{Conclusions}

In this work, different methodologies were applied, aiming to produce crosslinked PVA hydrogels for cartilage replacement, which besides presenting suitable mechanical and tribological properties, could be used as vehicles for the local delivery of the anti-inflammatories DFN or KTL.

CD hydrogels were produced and submitted to additional treatments: annealing, high hydrostatic pressure and $\gamma$ radiation. The resulting materials exhibited a dense non-porous structure, apart from HHP samples, which evidenced some porosity attributed to the breakdown of a few hydrogen bonds in the polymeric matrix induced by the pressurization treatment. These changes in the structure of HHP gels led to a higher water absorption capacity but did not affect their hydrophilicity. However, an accentuated decrease in the mechanical performance of the HHP material was observed, both in compression and tensile. Contrarily, annealing (especially at $150{ }^{\circ} \mathrm{C}$ ) and $\gamma$-irradiation 
decreased the hydrogel' swelling capacity and improved their mechanical resistance under tensile loads. This improvement was explained by the additional crosslinking and increase of hydrogel crystallinity induced by these treatments. Such changes had minor effects on the wettability of the materials. A150 samples presented the highest values of elastic modulus, strength, and toughness in both tensile and compression tests and revealed the most remarkable improvement in mechanical behavior.

Friction behavior of hydrogels tested in lubricated conditions against SS, in a ball-on-disc tribometer, under loads in the range of 20-60 N was distinctive for each type of material. HHP samples did not withstand any of the applied loads and GR gels only resisted the measurements at 20 and $30 \mathrm{~N}$. Annealing treatment led to a significant reduction of the friction. In particular, A150 samples showed the lowest values of CoF for loads between 20 and $40 \mathrm{~N}$ and were the only ones that withstand a load of $60 \mathrm{~N}$. Such behavior was associated with the greater mechanical performance of these materials, which may suffer less deformation and be more resistant to wear. Due to their superior performance, A150 samples were selected to be loaded with the anti-inflammatories. The release of the drugs was almost complete in the first $24 \mathrm{~h}$. The incorporation of Vit.E that creates hydrophobic barriers to drug diffusion improved the drug release profiles. Additional inclusion of a cationic surfactant CKC further sustained the release of the anionic drugs, especially of KTL, which shall be more dissociated. Irritability and cytotoxicity tests proved that A150 samples loaded with DFN or KTL and VitE. + CKC are safe from the biological point of view.

Here, we show that the annealing of PVA hydrogels at $150{ }^{\circ} \mathrm{C}$ can significantly improve their mechanical and tribological properties. More, these materials can be safely used as vehicles for local delivery of the anti-inflammatory drugs DFN or KTL. Therefore, A150 gels are promising candidates for load-bearing cartilage substitutes with a therapeutic effect in the immediate postoperative period. Further studies are required to address issues such as the integration of the material with the surrounding tissue and its fixation.

Supplementary Materials: The following are available online at http://www.mdpi.com/2075-4442/8/3/36/s1, Figure S1: Structures of polyvinyl alcohol (a), ketorolac tromethamine (b), and diclofenac sodium salt (c), with their respective $\mathrm{pKa}$ values, in water [1-3].

Author Contributions: Conceptualization: A.S.O. and A.P.S.; Methodology: A.S.O., S.S., P.N., I.B., and J.S.; Software: A.S.O. and S.S.; Validation: A.S.O. and S.S.; Formal analysis: A.S.O., R.C., and A.P.S.; Investigation: A.S.O.; Data curation: A.S.O. and S.S.; Writing-original draft preparation: A.S.O., P.N., and A.P.S.; Writing-review and editing: A.S.O., R.C., and A.P.S.; Supervision: R.C. and A.P.S.; Project administration: R.C. and A.P.S.; Funding acquisition: R.C. and A.P.S. All authors have read and agreed to the published version of the manuscript.

Funding: This research was funded by Fundação para a Ciência e a Tecnologia (FCT) through the Ph.D. grant PD/BD/128140/2016 (MIT-Portugal program) of A.S.O., the research project PTDC/CTM-CTM/29593/2017 and the unit projects UIDB/00100/2020 (CQE), UIDB/50022/2020 (LAETA, IDMEC), and UIDB/04585/2020 (CiiEM). S.S. received financial support from the Erasmus+ program, for the internship in BIOMAT Research Group at IST.

Acknowledgments: The authors gratefully acknowledge to Kuraray Co., Ltd. for providing the PVA powder used in this work and to Engineer Paula Matos and Engineer Nuno Inácio from Campus Tecnológico e Nuclear of Instituto Superior Técnico, Universidade de Lisboa for the irradiations with $\gamma$-rays.

Conflicts of Interest: The authors declare no conflict of interest.

\section{References}

1. Fox, A.J.S.; Bedi, A.; Rodeo, S.A. The basic science of articular cartilage: Structure, composition, and function. Sport Heal. A Multidiscip. Approach 2009, 1, 461-468.

2. Beddoes, C.; Whitehouse, M.; Briscoe, W.; Su, B. Hydrogels as a replacement material for damaged articular hyaline cartilage. Materials 2016, 9, 443. [CrossRef] [PubMed]

3. Rawal, B.R.; Ribeiro, R.; Chouksey, M.; Tripathi, K. Biomaterials for cartilage repair: A review. J. Med. Sci. 2013, 13, 615-620. [CrossRef]

4. Bicho, D.; Pina, S.; Reis, R.L.; Oliveira, J.M. Commercial products for osteochondral tissue repair and regeneration. In Osteochondral Tissue Engineering; Oliveira, J., Pina, S., Reis, R., San Roman, J., Eds.; Springer: Cham, Switzerland, 2018; Volume 1058, pp. 415-428. 
5. Chuang, E.-Y.; Chiang, C.-W.; Wong, P.-C.; Chen, C.-H. Hydrogels for the application of articular cartilage tissue engineering: A review of hydrogels. Adv. Mater. Sci. Eng. 2018, 2018, 1-13. [CrossRef]

6. Jagur-Grodzinski, J. Polymers for tissue engineering, medical devices, and regenerative medicine. Concise general review of recent studies. Polym. Adv. Technol. 2006, 17, 395-418. [CrossRef]

7. Święszkowski, W.; Ku, D.N.; Bersee, H.E.N.; Kurzydlowski, K.J. An elastic material for cartilage replacement in an arthritic shoulder joint. Biomaterials 2006, 27, 1534-1541. [CrossRef]

8. Oliveira, A.S.; Seidi, O.; Ribeiro, N.; Colaço, R.; Serro, A.P. Tribomechanical comparison between pva hydrogels obtained using different processing conditions and human cartilage. Materials 2019, 12, 3413. [CrossRef]

9. Baker, M.I.; Walsh, S.P.; Schwartz, Z.; Boyan, B.D. A review of polyvinyl alcohol and its uses in cartilage and orthopedic applications. J. Biomed. Mater. Res. Part B Appl. Biomater. 2012, 100, 1451-1457. [CrossRef]

10. Hassan, C.M.; Peppas, N.A. Structure and applications of poly (vinyl alcohol) hydrogels produced by conventional crosslinking or by freezing/thawing methods. In Biopolymers PVA Hydrogels, Anionic Polymerisation Nanocomposites; Springer: Heidelberg/Berlin, Germany, 2000; Volume 153, pp. 37-65.

11. Luo, X.; Akram, M.Y.; Yuan, Y.; Nie, J.; Zhu, X. Silicon dioxide/poly(vinyl alcohol) composite hydrogels with high mechanical properties and low swellability. J. Appl. Polym. Sci. 2019, 136, 46895. [CrossRef]

12. Zhou, D.; Dong, Q.; Liang, K.; Xu, W.; Zhou, Y.; Xiao, P. Photocrosslinked methacrylated poly (vinyl alcohol)/hydroxyapatite nanocomposite hydrogels with enhanced mechanical strength and cell adhesion. J. Polym. Sci. Part A Polym. Chem. 2019, 57, 1882-1889. [CrossRef]

13. Yang, L.; Wang, Z.; Fei, G.; Xia, H. Polydopamine particles reinforced poly (vinyl alcohol) hydrogel with nir light triggered shape memory and self-healing capability. Macromol. Rapid Commun. 2017, 38, 1700421. [CrossRef] [PubMed]

14. Zhang, Y.; Zhu, P.C.; Edgren, D. Crosslinking reaction of poly (vinyl alcohol) with glyoxal. J. Polym. Res. 2010, 17, 725-730. [CrossRef]

15. Park, J.-Y.; Hwang, K.-J.; Yoon, S.-D.; Lee, J.-H.; Lee, I.-H. Influence of glyoxal on preparation of poly (vinyl alcohol)/poly (acrylic acid) blend film. J. Nanosci. Nanotechnol. 2015, 15, 5955-5958. [CrossRef]

16. Peppas, N.A.; Hilt, J.Z.; Khademhosseini, A.; Langer, R. Hydrogels in biology and medicine: From molecular principles to bionanotechnology. Adv. Mater. 2006, 18, 1345-1360. [CrossRef]

17. Liu, T.; Jiao, C.; Peng, X.; Chen, Y.-N.; Chen, Y.; He, C.; Liu, R.; Wang, H. Super-strong and tough poly (vinyl alcohol)/poly (acrylic acid) hydrogels reinforced by hydrogen bonding. J. Mater. Chem. B 2018, 6, 8105-8114. [CrossRef]

18. Chen, K.; Chen, G.; Wei, S.; Yang, X.; Zhang, D.; Xu, L. Preparation and property of high strength and low friction PVA-HA/PAA composite hydrogel using annealing treatment. Mater. Sci. Eng. C 2018, 91, 579-588. [CrossRef] [PubMed]

19. Holloway, J.L.; Lowman, A.M.; Vanlandingham, M.R.; Palmese, G.R. Chemical grafting for improved interfacial shear strength in UHMWPE/PVA-hydrogel fiber-based composites used as soft fibrous tissue replacements. Compos. Sci. Technol. 2013, 85, 118-125. [CrossRef]

20. Jing, L.; Li, H.; Tay, R.Y.; Sun, B.; Tsang, S.H.; Cometto, O.; Lin, J.; Teo, E.H.T.; Tok, A.I.Y. Biocompatible hydroxylated boron nitride nanosheets/poly (vinyl alcohol) interpenetrating hydrogels with enhanced mechanical and thermal responses. ACS Nano 2017, 11, 3742-3751. [CrossRef]

21. Kobayashi, M.; Hyu, H.S. Development and evaluation of polyvinyl alcohol-hydrogels as an artificial articular cartilage for orthopedic implants. Materials 2010, 3, 2753-2771. [CrossRef]

22. Rejmontová, P.; Kovalcik, A.; Humpolíček, P.; Capáková, Z.; Wrzecionko, E.; Sáha, P. The use of fractionated Kraft lignin to improve the mechanical and biological properties of PVA-based scaffolds. RSC Adv. 2019, 9, 12346-12353. [CrossRef]

23. Suzuki, A.; Sasaki, S. Swelling and mechanical properties of physically crosslinked poly (vinyl alcohol) hydrogels. Proc. Inst. Mech. Eng. Part H J. Eng. Med. 2015, 229, 828-844. [CrossRef] [PubMed]

24. Sasaki, S.; Suzuki, A. Factors influencing the swelling and elution properties of poly (vinyl alcohol) cast gels. Polym. Adv. Technol. 2016, 27, 318-324. [CrossRef]

25. Negishi, J.; Nam, K.; Kimura, T.; Fujisato, T.; Kishida, A. High-hydrostatic pressure technique is an effective method for the preparation of PVA-heparin hybrid gel. Eur. J. Pharm. Sci. 2010, 41, 617-622. [CrossRef] [PubMed] 
26. Lian, Z.; Zhang, Y.; Zhao, Y. Nano-TiO2 particles and high hydrostatic pressure treatment for improving functionality of polyvinyl alcohol and chitosan composite films and nano-TiO2 migration from film matrix in food simulants. Innov. Food Sci. Emerg. Technol. 2016, 33, 145-153. [CrossRef]

27. Fukumori, T.; Nakaoki, T. Significant improvement of mechanical properties for polyvinyl alcohol film prepared from freeze/thaw cycled gel. Open J. Org. Polym. Mater. 2013, 3, 110-116. [CrossRef]

28. Gonzalez, J.S.; Alvarez, V.A. The effect of the annealing on the poly (vinyl alcohol) obtained by freezing-thawing. Thermochim. Acta 2011, 521, 184-190. [CrossRef]

29. Mondino, A.; González, M.; Romero, G.; Smolko, E. Physical properties of gamma irradiated poly (vinyl alcohol) hydrogel preparations. Radiat. Phys. Chem. 1999, 55, 723-726. [CrossRef]

30. Sasaki, S.; Omata, S.; Murakami, T.; Nagasawa, N.; Taguchi, M.; Suzuki, A. Effect of gamma ray irradiation on friction property of poly(vinyl alcohol) cast-drying on freeze-thawed hybrid gel. Gels 2018, 4, 30. [CrossRef]

31. Li, J.; Mooney, D.J. Designing hydrogels for controlled drug delivery. Nat. Rev. Mater. 2016, 1, 16071. [CrossRef]

32. Kou, L.; Xiao, S.; Sun, R.; Bao, S.; Yao, Q.; Chen, R. Biomaterial-engineered intra-articular drug delivery systems for osteoarthritis therapy. Drug Deliv. 2019, 26, 870-885. [CrossRef]

33. Rey-Rico, A.; Madry, H.; Cucchiarini, M. Hydrogel-based controlled delivery systems for articular cartilage repair. Biomed. Res. Int. 2016, 2016, 1215263. [CrossRef] [PubMed]

34. Mohammadi, F.; Mohammadi, S.S.; Tanideh, N.; Ahmadi, F. Hybrid scaffolds of hyaluronic acid and collagen loaded with prednisolone: An interesting system for osteoarthritis. Adv. Pharm. Bull. 2018, 8, 11-19. [CrossRef] [PubMed]

35. Bédouet, L.; Moine, L.; Pascale, F.; Nguyen, V.-N.; Labarre, D.; Laurent, A. Synthesis of hydrophilic intra-articular microspheres conjugated to ibuprofen and evaluation of anti-inflammatory activity on articular explants. Int. J. Pharm. 2014, 459, 51-61. [CrossRef] [PubMed]

36. Zhang, Z.; Bi, X.; Li, H.; Huang, G. Enhanced targeting efficiency of PLGA microspheres loaded with Lornoxicam for intra-articular administration. Drug Deliv. 2011, 18, 536-544. [CrossRef] [PubMed]

37. Kawadkar, J.; Chauhan, M.K. Intra-articular delivery of genipin cross-linked chitosan microspheres of flurbiprofen: Preparation, characterization, in vitro and in vivo studies. Eur. J. Pharm. Biopharm. 2012, 81, 563-572. [CrossRef] [PubMed]

38. Gunaydin, C.; Bilge, S.S. Effects of nonsteroidal anti-inflammatory drugs at the molecular level. Eurasian J. Med. 2018, 50, 116-121. [CrossRef] [PubMed]

39. Paradiso, P.; Galante, R.; Santos, L.; Alves de Matos, A.P.; Colaço, R.; Serro, A.P.; Saramago, B. Comparison of two hydrogel formulations for drug release in ophthalmic lenses. J. Biomed. Mater. Res. Part B Appl. Biomater. 2014, 102, 1170-1180. [CrossRef]

40. Filipe, H.P.; Henriques, J.; Reis, P.; Silva, P.C.; Quadrado, M.J.; Serro, A.P. Contact lenses as drug controlled release systems: A narrative review. Rev. Bras Oftalmol. 2016, 75, 241-247. [CrossRef]

41. Paradiso, P.; Serro, A.P.; Saramago, B.; Colaço, R.; Chauhan, A. Controlled release of antibiotics from vitamin e-loaded silicone-hydrogel contact lenses. J. Pharm. Sci. 2016, 105, 1164-1172. [CrossRef]

42. Topete, A. Effects of Sterilization on Drug Loaded Soft Contact Lenses. Ph.D. Thesis, Instituto Superior Técnico-University of Lisbon, Lisbon, Portugal, 2015.

43. Torres-Luna, C.; Hu, N.; Tammareddy, T.; Domszy, R.; Yang, J.; Wang, N.S.; Yang, A. Extended delivery of non-steroidal anti-inflammatory drugs through contact lenses loaded with Vitamin E and cationic surfactants. Contact Lens Anterior Eye 2019, 42, 546-552. [CrossRef]

44. ICCVAM. The hen's egg Test-Chorioallantoic membrane test method. In ICCVAM Test Method Evaluation Report: Current Validation Status of In Vitro Test Methods Proposed for Identifying Eye Injury Hazard Potential of Chemicals and Products; National Institute of Environmental Health Sciences: Research Triangle Park, NC, USA, 2010; pp. 19-25, NIH Publication No. 10-7553.

45. Oliveira, A. Effects of Sterilization on Drug Loaded Ophthalmic Lenses Materials. Ph.D. Thesis, Instituto Superior Técnico-University of Lisbon, Lisbon, Portugal, 2016.

46. Biological Evaluation of Medical Devices-Part 5: Tests for in Vitro Cytotoxicity (ISO 10993-5); International Organization for Standardization: Geneva, Switzerland, 2009.

47. Bandarra, S.; Mascarenhas, P.; Luís, A.R.; Catrau, M.; Bekman, E.; Ribeiro, A.C.; Félix, S.; Caldeira, J.; Barahona, I. In vitro and in silico evaluations of resin-based dental restorative material toxicity. Clin. Oral Investig. 2019. [CrossRef] [PubMed] 
48. Gulenoor, F.; Poddar, P. $\gamma$-irradiated polyvinyl alcohol (pva) and citric acid blend hydrogels: Swelling and absorption properties. Chem. Sci. J. 2016, 7, 1000125. [CrossRef]

49. Hassan, M.R.; Chowdhury, A.B.M.A.; Islam, M.T.; Poddar, P.; Dafader, N.C.; Chowdhury, A.M.S. Swelling and absorption properties of polyvinyl alcohol (pva) and acrylic acid blend hydrogels: Effect of of $\gamma$-irradiation. En Chem. Technol. Ind. J. 2016, 11, 107.

50. Zink, J.; Wyrobnik, T.; Prinz, T.; Schmid, M. Physical, chemical and biochemical modifications of protein-based films and coatings: An extensive review. Int. J. Mol. Sci. 2016, 17, 1376. [CrossRef]

51. Li, J.; Liu, H.; Wang, C.; Huang, G. A facile method to fabricate hybrid hydrogels with mechanical toughness using a novel multifunctional cross-linker. RSC Adv. 2017, 7, 35311-35319. [CrossRef]

52. Shi, Y.; Xiong, D.; Zhang, J. Effect of irradiation dose on mechanical and biotribological properties of PVA/PVP hydrogels as articular cartilage. Tribol. Int. 2014, 78, 60-67. [CrossRef]

53. Gaharwar, A.; Schexnailder, P.; Schmidt, G. Nanocomposite polymer biomaterials for tissue repair of bone and cartilage. In Nanobiomaterials Handbook; CRC Press: Boca Raton, EL, USA, 2011; pp. 1-28.

54. Cao, Z.; Wang, Y.; Wang, H.; Ma, C.; Li, H.; Zheng, J.; Wu, J.; Huang, G. Tough, ultrastretchable and tear-resistant hydrogels enabled by linear macro-cross-linker. Polym. Chem. 2019, 10, 3503-3513. [CrossRef]

55. Shi, F.-K.; Wang, X.-P.; Guo, R.-H.; Zhong, M.; Xie, X.-M. Highly stretchable and super tough nanocomposite physical hydrogels facilitated by the coupling of intermolecular hydrogen bonds and analogous chemical crosslinking of nanoparticles. J. Mater. Chem. B 2015, 3, 1187-1192. [CrossRef]

56. Li, W.; Wang, D.; Yang, W.; Song, Y. Compressive mechanical properties and microstructure of PVA-HA hydrogels for cartilage repair. RSC Adv. 2016, 6, 20166-20172. [CrossRef]

57. Pan, Y.-S.; Xiong, D.-S.; Ma, R.-Y. A study on the friction properties of poly (vinyl alcohol) hydrogel as articular cartilage against titanium alloy. Wear 2007, 262, 1021-1025. [CrossRef]

58. Pan, Y.S.; Xiong, D.S.; Chen, X.L. Friction characteristics of poly (vinyl alcohol) hydrogel as an articular cartilage biomaterial. Key Eng. Mater. 2007, 330-332, 1297-1300. [CrossRef]

59. Mow, V.C. The Role of Lubrication in Biomechanical Joints. J. Lubr. Technol. 1969, 91, 320-326. [CrossRef]

60. Neville, A.; Morina, A.; Liskiewicz, T.; Yan, Y. Synovial joint lubrication-Does nature teach more effective engineering lubrication strategies? Proc. Inst. Mech. Eng. Part C J. Mech. Eng. Sci. 2007, 221, 1223-1230. [CrossRef]

61. Li, F.; Su, Y.; Wang, J.; Wu, G.; Wang, C. Influence of dynamic load on friction behavior of human articular cartilage, stainless steel and polyvinyl alcohol hydrogel as artificial cartilage. J. Mater. Sci. Mater. Med. 2010, 21, 147-154. [CrossRef] [PubMed]

62. Forster, H.; Fisher, J. The influence of continuous sliding and subsequent surface wear on the friction of articular cartilage. Proc. Inst. Mech. Eng. H 1999, 213, 329-345. [CrossRef] [PubMed]

63. Brand, R.A. Joint contact stress: A reasonable surrogate for biological processes? Iowa Orthop. J. 2005, 25, 82-94. [PubMed]

64. Serro, A.P.; Colaço, R.; Saramago, B. Effect of albumin adsorption on biotribological properties of artificial joint materials. In Proteins at Interfaces III State of the Art; Horbett, T., Brash, J.L., Norde, W., Eds.; ACS Symposium Series: Washington, DC, USA, 2012; Volume 1120, pp. 497-523.

65. Ghalei, S.; Asadi, H.; Ghalei, B. Zein nanoparticle-embedded electrospun PVA nanofibers as wound dressing for topical delivery of anti-inflammatory diclofenac. J. Appl. Polym. Sci. 2018, 135, 46643. [CrossRef]

66. Peng, C.-C.; Kim, J.; Chauhan, A. Extended delivery of hydrophilic drugs from silicone-hydrogel contact lenses containing vitamin E diffusion barriers. Biomaterials 2010, 31, 4032-4047. [CrossRef]

67. Bengani, L.C.; Chauhan, A. Extended delivery of an anionic drug by contact lens loaded with a cationic surfactant. Biomaterials 2013, 34, 2814-2821. [CrossRef]

68. Barney, R.; Carroll, J.; Delaet, D. Surfactant studies of quaternary ammonium compounds: Critical surfactant concentration. J. Surfactants Deterg. 2006, 9, 137-140. [CrossRef]

69. Sinha, V.R.; Kumar, R.V.; Singh, G. Ketorolac tromethamine formulations: An overview. Expert Opin. Drug Deliv. 2009, 6, 961-975. [CrossRef] [PubMed]

70. Adeyeye, C.M.; Li, P.-K. Diclofenac sodium. In Analytical Profiles of Drug Substances; Academic Press: Cambridge, MA, USA, 1990; Volume 19, pp. 123-144.

71. Kalweit, S.; Gerner, I.; Spielmann, H. Validation project of alternatives for the draize eye test. Mol. Toxicol. 1987, 1, 597-603. [PubMed] 
72. Spielmann, H. HET-CAM test. In In Vitro Toxicity Testing Protocols. Methods in Molecular Biology ${ }^{T M}$; O'Hare, S., Atterwill, C.K., Eds.; Humana Press: Totowa, NJ, USA, 1995; pp. 199-204.

73. Eder, C.; Falkner, E.; Nehrer, S.; Losert, U.M.; Schoeffl, H. Introducing the concept of the 3Rs into tissue engineering research. ALTEX 2006, 23, 17-23. [PubMed]

74. Moreno-Jiménez, I.; Kanczler, J.M.; Hulsart-Billstrom, G.; Stefanie, I.; Oreffo, R.O.C. The chorioallantoic membrane assay for biomaterial testing in tissue engineering: A short-term in vivo preclinical model. Tissue Eng. Part C Methods 2017, 23, 938-952. [CrossRef]

75. Falkner, E.; Eder, C.; Kapeller, B.; Fröschl, W.; Schmatz, C.; Macfelda, K.; Losert, U.M. The mandatory cam testing of cells and scaffolds for tissue engineering: Benefits for the three RS of cooperation with the vaccine industry. Altern. Lab. Anim. 2004, 32, 573-580. [CrossRef]

(C) 2020 by the authors. Licensee MDPI, Basel, Switzerland. This article is an open access article distributed under the terms and conditions of the Creative Commons Attribution (CC BY) license (http://creativecommons.org/licenses/by/4.0/). 